TRANSACTIONS OF THE

AMERICAN MATHEMATICAL SOCIETY

Volume 352, Number 2, Pages 689-709

$\mathrm{S} 0002-9947(99) 02552-0$

Article electronically published on October 5, 1999

\title{
PRODUCTS AND DUALITY IN WALDHAUSEN CATEGORIES
}

\author{
MICHAEL S. WEISS AND BRUCE WILLIAMS
}

\begin{abstract}
The natural transformation $\Xi$ from $\mathbf{L}$-theory to the Tate cohomology of $\mathbb{Z} / 2$ acting on $\mathbf{K}$-theory commutes with external products. Corollary: The Tate cohomology of $\mathbb{Z} / 2$ acting on the $\mathbf{K}$-theory of any ring with involution is a generalized Eilenberg-Mac Lane spectrum, and it is 4 -periodic.
\end{abstract}

\section{INTRODUCTION}

Categories with cofibrations and weak equivalences (Waldhausen categories for short) were introduced in [Wald]. Generalizing earlier constructions due to Quillen and others, Waldhausen defined the $K$-theory spectrum $\mathbf{K}(\mathcal{C})$ of a Waldhausen category $\mathcal{C}$. It was realized early on [Vo] that suitable notions of Spanier-Whitehead duality in $\mathcal{C}$ give rise to involutions on $\mathbf{K}(\mathcal{C})$. We formalized this idea in [WWd] by introducing the notion of a Spanier-Whitehead product (SW product for short) in $\mathcal{C}$. To some extent this will be recalled below. When $\mathcal{C}$ is equipped with an SW product $\odot$ and $(\mathcal{C}, \odot)$ satisfies certain conditions $(\S 2$ of $[\mathrm{WWd}])$, then the following are defined:

1. a duality involution on $\mathbf{K}(\mathfrak{C})$ (more precisely, on something homotopy equivalent to $\mathbf{K}(\mathrm{C}))$;

2. a quadratic $L$-theory spectrum $\mathbf{L}_{\bullet}(\mathcal{C})$;

3. a symmetric $L$-theory spectrum $\mathbf{L} \bullet(\mathcal{C})$;

4. maps of spectra

$$
\begin{gathered}
\text { symmetrization }: \mathbf{L}_{\bullet}(\mathcal{C}) \longrightarrow \mathbf{L}^{\bullet}(\mathcal{C}), \\
\Xi: \mathbf{L}^{\bullet}(\mathcal{C}) \longrightarrow \mathbf{K}(\mathcal{C})^{t h \mathbb{Z} / 2} .
\end{gathered}
$$

Here $\mathbf{K}(\mathcal{C})^{t h \mathbb{Z} / 2}$ is the cofiber of the norm map from the homotopy orbit spectrum of the $\mathbb{Z} / 2$-action on $\mathbf{K}(\mathcal{C})$ to the homotopy fixed point spectrum:

$$
\mathbf{K}(\mathcal{C})_{h \mathbb{Z} / 2} \stackrel{\mathcal{N}}{\longrightarrow} \mathbf{K}(\mathcal{C})^{h \mathbb{Z} / 2} .
$$

(See [WW2, §2] and [AdCoDw] or [GreMa].) Our interest in the map $\Xi$, or rather in the composition of $\Xi$ with the symmetrization map from quadratic to symmetric $L$ theory, stems from the fact that it appears in an approximative algebraic description of structure spaces (moduli spaces of closed manifolds equipped with a homotopy equivalence to a fixed manifold $M$ ). Here we show that $\Xi$ commutes with external products - a fact which greatly facilitates calculations.

Received by the editors January 9, 1997.

1991 Mathematics Subject Classification. Primary 57N99, 57R50, 19D10.

Key words and phrases. Products, ring spectrum, Tate cohomology, surgery.

Both authors supported in part by NSF grant. 
0.1. Conventions, notation. Adopting a convention from [WWd], we usually denote spectra by boldface letters, and the corresponding (zero-th) infinite loop spaces by "unbold" letters. For example, $K(\mathcal{C})$ is an infinite loop space, and $\mathbf{K}(\mathcal{C})$ is a spectrum.

For the definition of spectrum and $C W$-spectrum, we refer to [Ad1]. We shall also need bispectra and $C W$-bispectra. A bispectrum is a family of pointed spaces $E_{m, n}$ for $m, n \in \mathbb{Z}$ and maps

$$
\begin{aligned}
\lambda_{m, n}: \Sigma E_{m, n} & \rightarrow E_{m+1, n}, \\
\rho_{m, n}: \Sigma E_{m, n} & \rightarrow E_{m, n+1}
\end{aligned}
$$

such that $\lambda_{m, n+1} \Sigma\left(\rho_{m, n}\right)=\rho_{m+1, n} \Sigma\left(\lambda_{m, n}\right) \tau: \Sigma^{2} E_{m, n} \rightarrow E_{m+1, n+1}$ where $\tau$ : $\Sigma^{2}$ ? $\rightarrow \Sigma^{2}$ ? permutes the suspension coordinates. If all $E_{m, n}$ are pointed $\mathrm{CW}-$ spaces and all $\lambda_{m, n}$ and $\rho_{m, n}$ are, up to CW-isomorphism, inclusions of CWsubspaces, then we speak of a CW-bispectrum. A map from a CW-bispectrum $\mathbf{X}$ to another bispectrum $\mathbf{Y}$ is defined as an equivalence class of pairs $\left(\mathbf{X}^{\prime}, f\right)$ where $\mathbf{X}^{\prime}$ is a cofinal CW-bi-subspectrum of $\mathbf{X}$ and $f: \mathbf{X}^{\prime} \rightarrow \mathbf{Y}$ is a so-called function. (This definition is completely analogous to Boardman's definition of a map from a CW-spectrum to another spectrum.)

Example/Definition. The smash product of a CW-spectrum $\mathbf{X}=\left\{X_{m}\right\}$ and a $\mathrm{CW}$-spectrum $\mathbf{Y}=\left\{Y_{n}\right\}$ is the $\mathrm{CW}$-bispectrum $\mathbf{X} \wedge \mathbf{Y}$ having the pointed $\mathrm{CW}$ space $X_{m} \wedge Y_{n}$ in bidegree $(m, n)$. (We have suppressed the structure maps for convenience of notation.) We realize that this is a very conservative approach to smash products, but for the purposes of this paper it seems just right.

\section{EXTERNAL PRODUCTS}

We assume that the reader is familiar with the $\mathcal{S}$. construction and the definition $K(\mathrm{C}):=\Omega\left|w \mathcal{S}_{\bullet} \mathrm{C}\right|$ from [Wald]. For reasons given in [Wald, $\left.\S 1.3\right], K(\mathrm{C})$ is an infinite loop space ("To pursue the analogy with Segal's version ... "). For external products, we quote directly from [Wald, §1.5]:

"We digress to indicate in which way the twice de-looped $K$-theory $w \mathcal{S}_{\bullet} \mathcal{S}_{\bullet} \mathcal{C}$ is used in defining products; or better, external pairings (products are induced from those). The ingredient that one needs is a bi-exact functor of categories with cofibrations and weak equivalences. This is a functor $\mathcal{A} \times \mathcal{B} \rightarrow \mathcal{C}, \quad(A, B) \mapsto A \wedge B$, having the property that for every $A \in \mathcal{A}$ and $B \in \mathcal{B}$ the partial functors $A \wedge$ ? and $? \wedge B$ are exact, and where in addition the following more technical condition must also be satisfied; namely, for every pair of cofibrations $A \mapsto A^{\prime}$ and $B \mapsto B^{\prime}$ in $\mathcal{A}$ and $\mathcal{B}$, respectively, the induced square of cofibrations in $\mathcal{C}$ must be admissible in the sense that the map $A^{\prime} \wedge B \cup_{A \wedge B} A \wedge B^{\prime} \rightarrow A^{\prime} \wedge B^{\prime}$ is a cofibration. A bi-exact functor induces a map, of bisimplicial bicategories

$$
w \mathcal{S}_{\bullet} \mathcal{A} \times w \mathcal{S}_{\bullet} \mathcal{B} \longrightarrow w w \mathcal{S}_{\bullet} \mathcal{S}_{\bullet} \mathcal{C}
$$

which upon passage to geometric realization factors through the smash product

$$
\left|w \mathcal{S}_{\bullet} \mathcal{A}\right| \wedge\left|w \mathcal{S}_{\bullet} \mathcal{B}\right| \longrightarrow\left|w w \mathcal{S}_{\bullet} \mathcal{S}_{\bullet} \mathcal{C}\right|
$$

and in turn induces

$$
\Omega\left|w \mathcal{S}_{\bullet} \mathcal{A}\right| \wedge \Omega\left|w \mathcal{S}_{\bullet} \mathcal{B}\right| \longrightarrow \Omega \Omega\left|w w \mathcal{S}_{\bullet} \mathcal{S}_{\bullet} \mathcal{C}\right|
$$

This is the desired pairing in $K$-theory in view of the homotopy equivalence of $\left|w \mathcal{S}_{\bullet} \mathcal{C}\right|$ with $\Omega\left|w \mathcal{S}_{\bullet} \mathcal{S}_{\bullet} \mathcal{C}\right|$, and a (much more innocent) homotopy equivalence of 
$w \delta_{.} \mathcal{S}_{.} \mathcal{C}$ with $w w \delta_{.} \mathcal{S}_{\bullet} \mathcal{C}$ which we will have occasion later on to consider in detail (the 'swallowing lemma'... )."

\section{External PRODUCTS AND SW PRODUCTS}

To begin, we recall from [WWd] the notion of an $\mathrm{SW}$ product. Let $\mathcal{C}$ be a Waldhausen category and let $*$ denote the zero object in $\mathcal{C}$.

2.1. Definition $[W W d, 1.1]$. By an $S W$ product on $\mathcal{C}$ we shall mean a functor

$$
(C, D) \mapsto C \odot D
$$

from $\mathcal{C} \times \mathcal{C}$ to the category of Spaces which is $w$-invariant, symmetric and bilinear (explanations follow).

- $w$-Invariance means that the functor takes pairs of weak equivalences to homotopy equivalences.

- Symmetry means that the functor comes with an isomorphism $\tau: C \odot D \cong$ $D \odot C$, natural in both variables, whose square is the identity on $C \odot D$.

- Bilinearity means (in the presence of symmetry) that, for fixed but arbitrary $D$, the functor $C \mapsto C \odot D$ takes any cofiber square in $\mathcal{C}$ to a homotopy pullback square of Spaces. (A cofiber square is a commutative pushout square in which either the horizontal or the vertical arrows are cofibrations.) Bilinearity also means that $* \odot D$ is contractible.

- The category of Spaces (with a capital S) refers to the category of fibrant simplicial sets. (This is mostly to ensure that we have canonically defined homotopy inverse limits in our category of Spaces.)

If $C \odot D$ is naturally pointed for all $C$ and $D$, and $\tau: C \odot D \rightarrow D \odot C$ is a pointed map, then $\odot$ will be called a pointed SW product. Every SW product $\odot$ can be upgraded to a pointed SW product as follows: first replace $C \odot D$ by the homotopy equivalent $(C \odot D) /(* \odot *)$, which is pointed, and then apply Kan's natural procedure for making arbitrary simplicial sets fibrant.

Let $\mathcal{H} \mathcal{C}$ be the category of fractions obtained from $\mathcal{C}$ by making all weak equivalences in $\mathcal{C}$ invertible. (We assume that $\mathcal{C}$ is small or at least equivalent to a small category.) Write $[C, D]$ for the set of morphisms from $C$ to $D$ in $\mathcal{H C}$. The conditions or axioms that we impose on $(\mathcal{C}, \odot)$ to ensure that things like $\mathbf{L}_{\bullet}(\mathcal{C}), \mathbf{L}^{\bullet}(\mathcal{C})$, $\mathbf{K}(\mathcal{C})^{t h \mathbb{Z} / 2}$ and the $\Xi$-map exist are briefly as follows [WWd, $\S 2$ ].

i) $\mathcal{C}$ admits a cylinder functor satisfying the cylinder axiom.

ii) $\mathcal{H}$ is stable, i.e., the suspension $\Sigma: \mathcal{H C} \rightarrow \mathcal{H C}$ is an equivalence of categories.

iii) A morphism in $\mathcal{C}$ is a weak equivalence if it becomes an isomorphism in $\mathcal{H C}$.

iv) For each $B$ in $\mathcal{H}$, the functor on $\mathcal{H C}$ given by $C \mapsto \pi_{0}(B \odot C)$ is corepresentable, say by an object $T(B)$. (Note that $T$ can be regarded as a functor $\mathcal{H} \mathcal{C}^{\text {op }} \rightarrow \mathcal{H} \mathcal{H} ;$ it is self-adjoint since $[T(B), C] \cong \pi_{0}(B \odot C) \cong \pi_{0}(C \odot B) \cong$ $[T(C), B]$.

v) The canonical morphism $T^{2}(B) \rightarrow B$ (adjoint to id :T(B) $\rightarrow T(B)$ ) is an isomorphism for every $B$ in $\mathcal{H}$.

We say that an element in $\pi_{0}(B \odot C)$ is nondegenerate if the corresponding morphism $T(B) \rightarrow C$ in $\mathcal{H C}$ is an isomorphism. More generally, an element in $\pi_{k}(B \odot C)$ will be considered nondegenerate if the corresponding element in $\pi_{0}\left(\Sigma^{-k} B \odot C\right)$ is nondegenerate. Condition $\mathrm{v}$ ) ensures that $\tau$ takes nondegenerate elements in $\pi_{k}(B \odot C)$ to nondegenerate elements in $\pi_{k}(C \odot B)$. 
2.2. Outline. In sections 3,4 and 5 below we work with three Waldhausen categories $\mathcal{A}, \mathcal{B}$ and $\mathcal{C}$ related by a bi-exact functor $(A, B) \mapsto A \diamond B$ from $\mathcal{A} \times \mathcal{B}$ to $\mathcal{C}$. In addition we assume that $\mathcal{A}, \mathcal{B}$ and $\mathcal{C}$ are equipped with pointed $\mathrm{SW}$ products satisfying the usual axioms and that the functor $(A, B) \rightarrow A \diamond B$ intertwines the $\mathrm{SW}$ products (details follow). The short-term goal is then to produce maps of spaces, from $L^{\bullet}(\mathcal{A}) \wedge L^{\bullet}(\mathcal{B})$ to $L^{\bullet}(\mathcal{C})$ and from $\Omega^{\infty}\left(\mathbf{K}(\mathcal{A})^{t h \mathbb{Z} / 2}\right) \wedge \Omega^{\infty}\left(\mathbf{K}(\mathcal{B})^{t h \mathbb{Z} / 2}\right)$ to $\Omega^{\infty}\left(\mathbf{K}(\mathcal{C})^{t h \mathbb{Z} / 2}\right)$, reflecting the category theoretic data at the space level. Here $L^{\bullet}(\mathcal{A})$ is short for $\Omega^{\infty} \mathbf{L}^{\bullet}(\mathcal{A})$, and so on. Most important for us, we want commutativity in the diagram

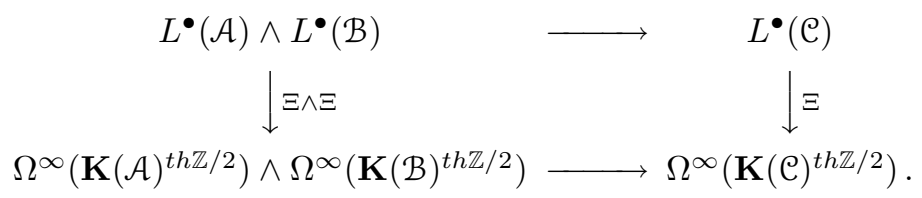

Possible and interesting choices for $\mathcal{A}, \mathcal{B}$ and $\mathcal{C}$ etc. are listed in $\S 2$.A. In $\S 6$ we define the products at the spectrum level and establish multiplicativity at the spectrum level. Some more input is required for that, but for now the following suffices.

2.3. Hypotheses in detail. We assume that $\mathcal{A}, \mathcal{B}$ and $\mathcal{C}$ are equipped with $\mathrm{SW}$ products, all denoted by $\odot$ for economy. In addition to the bi-exact functor $\diamond$ : $\mathcal{A} \times \mathcal{B} \rightarrow \mathcal{C}$ we need a natural map of simplicial sets, the "intertwiner":

$$
\jmath:\left(A_{1} \odot A_{2}\right) \wedge\left(B_{1} \odot B_{2}\right) \rightarrow\left(A_{1} \diamond B_{1}\right) \odot\left(A_{2} \diamond B_{2}\right)
$$

for $A_{1}, A_{2}$ in $\mathcal{A}$ and $B_{1}, B_{2}$ in $\mathcal{B}$. These data are subject to three rather obvious conditions. The first is that axioms i) $-\mathrm{v}$ ) above must hold for $(\mathcal{A}, \odot)$ and $(\mathcal{B}, \odot)$ and $(\mathrm{C}, \odot)$. The second condition is that the diagram

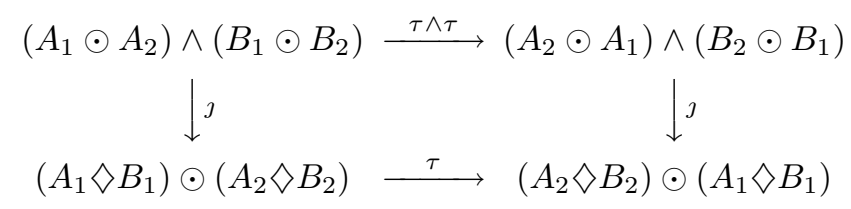

must commute for $A_{1}, A_{2}$ in $\mathcal{A}$ and $B_{1}, B_{2}$ in $\mathcal{B}$. Third condition: If $[\eta] \in$ $\pi_{0}\left(A_{1} \odot A_{2}\right)$ and $[\zeta] \in \pi_{0}\left(B_{1} \odot B_{2}\right)$ are nondegenerate [WWd, 3.6], then $[\jmath(\eta \wedge \zeta)]$ is nondegenerate in $\pi_{0}\left(\left(A_{1} \diamond B_{1}\right) \odot\left(A_{2} \diamond B_{2}\right)\right)$.

\section{A. ExAmples}

2.A.1. Preliminaries. Let $E \mapsto E^{\sharp}$ be the Kan-Dold functor $[\mathrm{Cu}]$ from chain complexes to simplicial abelian groups ; the $n$-simplices of $E^{\sharp}$ are the chain maps from the cellular chain complex of the CW-space $\Delta^{n}$ to $E$.

The Kan-Dold functor is not well-behaved with respect to tensor products. To repair this we introduce another functor $E \mapsto E^{\sharp \sharp}$ from chain complexes to bisimplicial abelian groups ; the $(m, n)$-bisimplices of $E^{\sharp \sharp}$ are the chain maps from the cellular chain complex of the product CW-space $\Delta^{m} \times \Delta^{n}$ to $E$. Note that the set of $(m, n)$-bisimplices is in canonical bijection with the set of $(n, m)$-bisimplices ; this leads to a canonical involution on $\left|E^{\sharp \sharp}\right|$ and also to a canonical involution on the diagonal of $E^{\sharp \sharp}$ (a simplicial set whose $n$-simplices are the $(n, n)$-bisimplices of $E^{\sharp \sharp}$, and whose realization is homeomorphic to that of $\left.E^{\sharp \sharp}\right)$.

A natural bisimplicial map $E^{\sharp} \times F^{\sharp} \rightarrow(E \otimes F)^{\sharp \sharp}$ is given by $(f, g) \mapsto f \otimes g$ for an $m$-simplex $f \in E^{\sharp}$, and an $n$-simplex $g \in F^{\sharp}$. Note that this is not a 
homomorphism. It factors through the smash product $E^{\sharp} \wedge F^{\sharp}$. We may regard it as a map of simplicial sets by passing to diagonals.

2.A.2. Example. Given rings with involution $R$ and $S$, let $\mathcal{A}, \mathcal{B}$ and $\mathcal{C}$ be the categories of bounded chain complexes of f.g. projective left modules over $R, S$ and $T=R \otimes S$, respectively. Define the SW product on $\mathcal{A}, \mathcal{B}$ and $\mathcal{C}$ by

$$
C \odot D:=\left\{\begin{array}{l}
\left(C \otimes_{R} D\right)^{\sharp} \\
\left(C \otimes_{S} D\right)^{\sharp} \\
\left(C \otimes_{T} D\right)^{\sharp}
\end{array}\right.
$$

respectively. Define $\diamond: \mathcal{A} \times \mathcal{B} \rightarrow \mathcal{C}$ by $C \diamond D:=C \otimes D$. The definition of $\tau: C \odot D \rightarrow$ $D \odot C$ is obvious for $C, D$ in $\mathcal{A}$ or $\mathcal{B}$. For $C, D$ in $\mathcal{C}$ we define $\tau: C \odot D \rightarrow D \odot C$ by combining $C \otimes_{T} D \rightarrow D \otimes_{T} C$ with the canonical involution on ?\#\#.

The "intertwiner" $\jmath:(C \odot D) \wedge\left(C^{\prime} \odot D^{\prime}\right) \rightarrow\left(C \diamond C^{\prime}\right) \odot\left(D \diamond D^{\prime}\right)$ for $C, D$ in $\mathcal{A}$ and $C^{\prime}, D^{\prime}$ in $\mathcal{B}$ is obtained by writing the domain as $E^{\sharp} \wedge F^{\sharp}$ and the codomain as $(E \otimes F)^{\sharp \sharp}$, for $E=C \otimes_{R} D$ and $F=C^{\prime} \otimes_{S} D^{\prime}$, and applying the construction of 2.A.1. The conditions in 2.3 are satisfied.

2.A.3. Example. Let $\mathcal{A}$ be the category of $\mathrm{CW}$-spectra (in the sense of Boardman, [Ad1]) with finitely many cells, with cellular maps as morphisms. Let $\mathcal{B}=\mathcal{A}$ and let $\mathcal{C}$ be the category of $\mathrm{CW}$-bispectra with finitely many cells. We regard the smash product of two CW-spectra as a CW-bispectrum. In this spirit, define $\diamond: \mathcal{A} \times \mathcal{B} \rightarrow \mathcal{C}$ by $\mathbf{X} \diamond \mathbf{Y}:=\mathbf{X} \wedge \mathbf{Y}$ and define $\odot$ on $\mathcal{A}, \mathcal{B}$ by

$$
\mathbf{X} \odot \mathbf{Y}:=\Omega^{\infty}(\mathbf{X} \wedge \mathbf{Y})
$$

(the simplicial set whose $n$-simplices are the bispectrum maps $\Delta_{+}^{n} \wedge \mathbf{S}^{0} \wedge \mathbf{S}^{0} \rightarrow$ $\mathbf{X} \wedge \mathbf{Y}$ ). For $\mathbf{X}, \mathbf{Y}$ in $\mathcal{C}$ let $\mathbf{X} \odot \mathbf{Y}$ be the diagonal of the bisimplicial set whose $(m, n)$-bisimplices are the 4-spectrum maps

$$
\Delta_{+}^{m} \wedge \Delta_{+}^{n} \wedge \mathbf{S}^{0} \wedge \mathbf{S}^{0} \wedge \mathbf{S}^{0} \wedge \mathbf{S}^{0} \longrightarrow \mathbf{X} \wedge \mathbf{Y}
$$

The intertwiner $\jmath$ is defined (modulo obvious identifications, as a map of simplicial sets) by $(f, g) \mapsto f \wedge g$ for $m$-simplices $f \in \mathbf{X} \odot \mathbf{Y}$ and $g \in \mathbf{X}^{\prime} \odot \mathbf{Y}^{\prime}$, with $\mathbf{X}$, $\mathbf{Y}$ in $\mathcal{A}$ and $\mathbf{X}^{\prime}, \mathbf{Y}^{\prime}$ in $\mathcal{B}$. The definition of $\tau$ is obvious in the case of $\mathcal{A}$ and $\mathcal{B}$, and it is analogous to that in 2.A.2 in the case of $\mathcal{C}$. The conditions in 2.3 are satisfied.

2.A.4. Example. Given $\mathcal{A}, \mathcal{B}$ and $\mathcal{C}$ with $\diamond$ and $\mathrm{SW}$ products and intertwiner $\jmath$ as in 2.3, we can generate more examples by parametrization. Let $X$ and $Y$ be finite simplicial complexes. Let $\mathcal{A}^{*}(X)$ be the category of colimit-preserving functors from the poset of subcomplexes of $X$ to $\operatorname{cof} \mathcal{A}$. See [WWd, §1.5] for more details. The functors in question are determined up to unique isomorphism by their restriction to the poset of faces of $X$, and for this reason it is permitted to think of $\mathcal{A}^{*}(X)$ as a category of certain functors from the poset of faces of $X$ to $\mathcal{A}$. We shall do so. We view $\mathcal{B}^{*}(Y)$ in the same way, and think of $\mathcal{C}^{*}(X, Y):=\left(\mathcal{C}^{*}(X)\right)^{*}(Y)$ as a category of certain functors from the poset of pairs $(s, t)$ to $\mathcal{C}$. Here $s$ and $t$ are faces of $X$ and $Y$, respectively. Now we can extend $\diamond: \mathcal{A} \times \mathcal{B} \rightarrow \mathcal{C}$ to $\diamond: \mathcal{A}^{*}(X) \times \mathcal{B}^{*}(Y) \rightarrow \mathcal{C}^{*}(X, Y)$ by the rule $\left(F_{1} \diamond F_{2}\right)(s, t):=F_{1}(s) \diamond F_{2}(t)$. The intertwiner $\jmath$ also extends. To see this we recall that an SW product on $\mathcal{A}^{*}(X)$ is defined in terms of that on $\mathcal{A}$ by the formula

$$
F_{1} \odot F_{2}:=\operatorname{holim}_{s} F_{1}(s) \odot F_{2}(s)
$$


for $F_{1}, F_{2}$ in $\mathcal{A}^{*}(X)$. We use the same formula to equip $\mathcal{B}^{*}(Y)$ with an SW product; in $\mathcal{C}^{*}(X, Y)$ we must define

$$
F_{1} \odot F_{2}:=\operatorname{holim}_{s, t} F_{1}(s, t) \odot F_{2}(s, t)
$$

where $s, t$ run through the faces of $\Delta^{m}$ and $\Delta^{n}$, respectively. So if $F_{1}=E_{1} \diamond E_{1}^{\prime}$ and $F_{2}=E_{2} \diamond E_{2}^{\prime}$, then $F_{1} \odot F_{2}$ becomes

$$
\operatorname{holim}_{s, t}\left(E_{1}(s) \diamond E_{1}^{\prime}(t)\right) \odot\left(E_{2}(s) \diamond E_{2}^{\prime}(s)\right)
$$

and as such receives a canonical map, induced by $\jmath$, from

$$
\underset{s, t}{\operatorname{holim}}\left(E_{1}(s) \odot E_{2}(s)\right) \wedge\left(E_{1}^{\prime}(t) \wedge E_{2}^{\prime}(t)\right)
$$

which in turn receives a canonical map from

$$
\left[\operatorname{holim}_{s}\left(E_{1}(s) \odot E_{2}(s)\right)\right] \wedge\left[\operatorname{holim}_{t}\left(E_{1}^{\prime}(t) \odot E_{2}^{\prime}(t)\right)\right],
$$

that is, from $\left(E_{1} \odot E_{2}\right) \wedge\left(E_{1}^{\prime} \odot E_{2}^{\prime}\right)$. The composite map

$$
\left(E_{1} \odot E_{2}\right) \wedge\left(E_{1}^{\prime} \odot E_{2}^{\prime}\right) \longrightarrow\left(E_{1} \diamond E_{1}^{\prime}\right) \odot\left(E_{2} \diamond E_{2}^{\prime}\right)
$$

is the intertwiner we are looking for: a natural transformation between functors from $\mathcal{A}^{*}(X) \times \mathcal{A}^{*}(X) \times \mathcal{B}^{*}(Y) \times \mathcal{B}^{*}(Y)$ to pointed Spaces. Let us call it $\bar{\jmath}$, temporarily, to distinguish it from the original $\jmath$. The conditions in 2.3 are satisfied, and here it is worth checking the third (on nondegeneracy). Suppose therefore that $[\eta] \in \pi_{0}\left(E_{1} \odot E_{2}\right)$ and $[\zeta] \in \pi_{0}\left(E_{1}^{\prime} \odot E_{2}^{\prime}\right)$ are nondegenerate. By [WWd, 8.8] nondegeneracy of $[\eta]$ can be expressed as follows: for each face $s \subset X$, the image $\left[\eta_{s}\right]$ of $[\eta]$ under the specialization map

$$
\pi_{0}\left(E_{1} \odot E_{2}\right) \rightarrow \pi_{|s|}\left(E_{1}(s) \odot E_{2}(s / \partial s)\right)
$$

is nondegenerate. Here $E_{2}(s / \partial s)$ is short for $E_{2}(s) / E_{2}(\partial s)$. Nondegeneracy of $[\zeta]$ can be expressed in the same way. A twofold application of [WWd, 8.8] shows that $[\xi]:=[\bar{\jmath}(\eta \wedge \zeta)]$ is nondegenerate provided its image $\left[\xi_{s t}\right]$ under

$\pi_{0}\left(\left(E_{1} \diamond E_{1}^{\prime}\right) \odot\left(E_{2} \diamond E_{2}^{\prime}\right)\right) \longrightarrow \pi_{|s|+|t|}\left(\left(E_{1} \diamond E_{1}^{\prime}\right)(s \times t) \odot\left(E_{2} \diamond E_{2}^{\prime}\right)((s \times t) / \partial(s \times t))\right)$

is nondegenerate, for every face $s \subset X$ and every face $t \subset Y$. By rewriting the codomain as $\left(E_{1}(s) \diamond E_{1}^{\prime}(t)\right) \odot\left(E_{2}(s / \partial s) \diamond E_{2}^{\prime}(t / \partial t)\right)$, we see that $\left[\xi_{s t}\right]=\left[\jmath\left(\eta_{s} \wedge \zeta_{t}\right)\right]$, which is indeed nondegenerate because of our assumption on $\jmath$.

2.A.5. Example. Again suppose that $\mathcal{A}, \mathcal{B}$ and $\mathcal{C}$ are given as in 2.3 with $\diamond$ and $\mathrm{SW}$ products and intertwiner $\jmath$. We note that $\diamond$ extends to a bi-exact functor

$$
\mathcal{S}_{k} \mathcal{A} \times \mathcal{S}_{\ell} \mathcal{B} \rightarrow \mathcal{S}_{k} \mathcal{S}_{\ell} \mathcal{C}
$$

by $(E \diamond F)\left(i, j, i^{\prime}, j^{\prime}\right):=E(i, j) \diamond F\left(i^{\prime}, j^{\prime}\right)$. See [WWd, $\left.\S 1\right]$ for notation. The appropriate $\mathrm{SW}$ products on $\mathcal{S}_{k} \mathcal{A}, \mathcal{S}_{\ell} \mathcal{B}$ and $\mathcal{S}_{k} \mathcal{S}_{\ell} \mathcal{C}$ are also described in [WWd, 1 ] ; they are given by

$$
\begin{gathered}
A_{1} \odot A_{2}:=\operatorname{holim} A_{1}(i, j) \odot A_{2}(p, q), \\
B_{1} \odot B_{2}:=\operatorname{holim} B_{1}\left(i^{\prime}, j^{\prime}\right) \odot B_{2}\left(p^{\prime}, q^{\prime}\right), \\
C_{1} \odot C_{2}:=\operatorname{holim} C_{1}\left(i, j, i^{\prime}, j^{\prime}\right) \odot C_{2}\left(p, q, p^{\prime}, q^{\prime}\right)
\end{gathered}
$$

for $A_{1}, A_{2}$ in $\mathcal{S}_{k} \mathcal{A}$ and $B_{1}, B_{2}$ in $\mathcal{S}_{\ell} \mathcal{B}$ and $C_{1}, C_{2}$ in $\mathcal{S}_{k} \mathcal{S}_{\ell} \mathcal{C}$, with the following conditions on the indices: $i+q$ and $j+p$ are $\geq k$, whereas $i^{\prime}+q^{\prime}$ and $j^{\prime}+p^{\prime}$ are $\geq \ell$. If $C_{1}=A_{1} \diamond B_{1}$ and $C_{2}=A_{2} \diamond B_{2}$ then 
$\jmath:\left(A_{1}(i, j) \odot A_{2}(p, q)\right) \wedge\left(\left(B_{1}\left(i^{\prime}, j^{\prime}\right) \odot B_{2}\left(p^{\prime}, q^{\prime}\right)\right) \longrightarrow C_{1}\left(i, j, i^{\prime}, j^{\prime}\right) \odot C_{2}\left(p, q, p^{\prime}, q^{\prime}\right)\right.$

is a natural transformation of functors in the variable $\left(i, j, i^{\prime}, j^{\prime}, p, q, p^{\prime}, q^{\prime}\right)$ and induces therefore

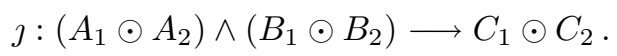

Again the conditions in 2.3 are satisfied for $\mathcal{S}_{k} \mathcal{A}, \mathcal{S}_{\ell} \mathcal{B}$ and $\mathcal{S}_{k} \mathcal{S}_{\ell} \mathcal{C}$ with the extended SW products and the extended $\diamond$ and $\jmath$. In particular, the nondegeneracy condition can be verified using [WWd, 6.6]. We omit the details.

\section{Products at the SPace LeVel: Symmetric $L$-Theory}

Fix $\mathcal{A}, \mathcal{B}, \mathcal{C}$ and additional structure as in 2.3. Define the $\Delta$-set $L^{\bullet}(\mathcal{A})$ in accordance with [WWd, §9], so that the set of $m$-simplices is the set of 0 -dimensional symmetric Poincaré objects in $\mathcal{A}^{*}(m)=\mathcal{A}^{*}\left(\Delta^{m}\right)$. Define $L^{\bullet}(\mathcal{B})$ in the same manner. It is clear that we need a description of $L^{\bullet}(\mathcal{C})$ as a bi- $-{ }^{-}$-set if we want to have a product map $L^{\bullet}(\mathcal{A}) \wedge L^{\bullet}(\mathcal{B}) \rightarrow L^{\bullet}(\mathcal{C})$. We therefore re-define $L^{\bullet}(\mathcal{C})$ as the bi$\Delta$-set whose $(m, n)$-bisimplices are the 0 -dimensional symmetric Poincaré objects in $\mathcal{C}^{*}(m, n):=\mathcal{C}^{*}\left(\Delta^{m}, \Delta^{n}\right)$ (see example 2.A.4). To legalize the inflated definition of $L \bullet(\mathcal{C})$ we observe that it is isomorphic to the realization of the $\Delta$-space

$$
m \mapsto L^{\bullet}\left(\mathfrak{C}^{*}(m)\right)
$$

with the traditional definition of $L^{\bullet}\left(\mathrm{C}^{*}(m)\right)$. Each $L^{\bullet}\left(\mathrm{C}^{*}(m)\right)$ is a fibrant $\Delta$-set by the remark following [WWd, 9.2], and comparison of the homotopy groups shows that all face operators $L^{\bullet}\left(\mathfrak{C}^{*}\left(m_{1}\right)\right) \rightarrow L^{\bullet}\left(\mathrm{C}^{*}\left(m_{2}\right)\right)$ in $(\bullet)$ are homotopy equivalences. Hence the geometric realization of $(\bullet)$ has the correct homotopy type. We are now ready for the product map $L^{\bullet}(\mathcal{A}) \wedge L^{\bullet}(\mathcal{B}) \rightarrow L^{\bullet}(\mathcal{C})$, as a map between bi- $\Delta$-sets (see the remark just below). A typical $(m, n)$-bisimplex in $L^{\bullet}(\mathcal{A}) \times L^{\bullet}(\mathcal{B})$ is a pair consisting of a 0-dimensional symmetric Poincaré object $(A, \phi)$ in $\mathcal{A}^{*}(m)$ and a 0 -dimensional symmetric Poincaré object $(B, \psi)$ in $\mathcal{B}^{*}(n)$. Using 2.A.4, we map this to the $(m, n)$-bisimplex in $L^{\bullet}(\mathcal{C})$ given by the object $A \diamond B$ in $\mathcal{C}^{*}(m, n)$ together with the 0 -dimensional symmetric Poincaré structure

$$
\jmath(\phi \wedge \psi) \in((A \diamond B) \odot(A \diamond B))^{h \mathbb{Z} / 2} .
$$

Remark. $L^{\bullet}(\mathcal{A})$ and $L^{\bullet}(\mathcal{B})$ are pointed $\Delta$-sets, i.e., contravariant functors from a certain category $\Delta$ to the category of pointed sets. The product of two $\Delta$-sets can be defined roughly like the product of two simplicial sets, but products in this sense do not commute with geometric realization. A similar remark applies to smash products. We therefore prefer to define the smash product of two pointed $\Delta$-sets $X, Y$ as the bi- $\Delta$-set which in bidegree $(m, n)$ equals the pointed set $X_{m} \wedge Y_{n}$. Smash product in this sense commutes with geometric realization, provided the geometric realizations are taken in the pointed sense: all (bi)-simplices corresponding to a base point, in any degree or bidegree, must be collapsed.

\section{Products at the space level: K-Theory and the Tate construction}

We keep the notation of the preceding section. From $\S 1$ we know that $\diamond$ : $\mathcal{A} \times \mathcal{B} \rightarrow \mathcal{C}$ gives rise to a map $\Omega\left|w \mathcal{S}_{\bullet} \mathcal{A}\right| \wedge \Omega\left|w \mathcal{S}_{\bullet} \mathcal{B}\right| \longrightarrow \Omega \Omega\left|w w \mathcal{S}_{\bullet} \mathcal{S}_{\bullet} \mathcal{C}\right|$ which we can write informally as $K(\mathcal{A}) \wedge K(\mathcal{B}) \rightarrow K(\mathcal{C})$. What we would like to have, though, is a product map from $\Omega^{\infty}\left(\mathbf{K}(\mathcal{A})^{t h \mathbb{Z} / 2}\right) \wedge \Omega^{\infty}\left(\mathbf{K}(\mathcal{B})^{t h \mathbb{Z} / 2}\right)$ to $\Omega^{\infty}\left(\mathbf{K}(\mathcal{C})^{t h \mathbb{Z} / 2}\right)$, described in a way which makes the comparison with product maps in symmetric 
$L$-theory easy. We start by describing the appropriate models for $\Omega^{\infty}$ of $\mathbf{K}(\mathcal{A})^{\operatorname{th} \mathbb{Z} / 2}$, $\mathbf{K}(\mathcal{B})^{t h \mathbb{Z} / 2}$ and $\mathbf{K}(\mathcal{C})^{t h \mathbb{Z} / 2}$. Our model for $\Omega^{\infty}\left(\mathbf{K}(\mathcal{A})^{t h \mathbb{Z} / 2}\right)$ is the geometric realization of $m \mapsto K\left(\mathcal{A}^{*}(m)\right)^{h \mathbb{Z} / 2}$ as in [WWd, $\S 9$ ]. Since this involves spaces $K\left(\mathcal{A}^{*}(m)\right)$ for $m \geq 0$, we must add that the preferred model for $K\left(\mathcal{A}^{*}(m)\right)$ is $\Omega\left|x w \mathcal{S}_{\bullet} \mathcal{A}^{*}(m)\right|$, defined as in [WWd, §7]. The $x$ prefix indicates a modification of Waldhausen's $\mathcal{S}$. construction which depends on the SW product. With these models, $\mathbb{Z} / 2$ acts on each $K\left(\mathcal{A}^{*}(m)\right)$ so that $K\left(\mathcal{A}^{*}(m)\right)^{h \mathbb{Z} / 2}$ has a meaning. - The preferred model for $\Omega^{\infty}\left(\mathbf{K}(\mathcal{B})^{t h \mathbb{Z} / 2}\right)$ is constructed in the same manner. As we shall explain, an appropriate model for $\Omega^{\infty}\left(\mathbf{K}(\mathrm{C})^{t h \mathbb{Z} / 2}\right)$ is the geometric realization of the bi- $\Delta^{-}$ space $(m, n) \mapsto K\left(\mathcal{C}^{*}(m, n)\right)^{h \mathbb{Z} / 2}$, with $\mathcal{C}^{*}(m, n)$ as in the preceding section. Here $K\left(\mathrm{C}^{*}(m, n)\right)$ itself must be defined as $\Omega \Omega\left|x w w \mathcal{S}_{\bullet} \mathcal{S}_{\bullet} \mathcal{C}^{*}(m, n)\right|$. Again the prefix $x$ is there to ensure that $\mathbb{Z} / 2$ acts. To verify that $\left|(m, n) \mapsto K\left(\mathcal{C}^{*}(m, n)\right)^{h \mathbb{Z} / 2}\right|$ has the right homotopy type, i.e. that of $\Omega^{\infty}\left(\mathbf{K}(\mathcal{C})^{t h \mathbb{Z} / 2}\right)$, what we need to show is that the face operators in the $\Delta$-space

$$
m \mapsto\left|n \mapsto K\left(\mathcal{C}^{*}(m, n)\right)^{h \mathbb{Z} / 2}\right|
$$

are all homotopy equivalences. Since $\left|n \mapsto K\left(\mathcal{C}^{*}(m, n)\right)^{h \mathbb{Z} / 2}\right|$ for fixed $m$ is related to $\Omega^{\infty}\left(\mathbf{K}\left(\mathcal{C}^{*}(m)\right)^{t h \mathbb{Z} / 2}\right)$ by a chain of natural homotopy equivalences, we are reduced to showing that the face operators in

$$
m \mapsto \mathbf{K}\left(\mathcal{C}^{*}(m)\right)^{t h \mathbb{Z} / 2}
$$

are all homotopy equivalences. But that is clear because the mapping cone of any face map $\mathbf{K}\left(\mathcal{C}^{*}(m)\right) \rightarrow \mathbf{K}\left(\mathcal{C}^{*}(0)\right)$ is induced as a spectrum with $\mathbb{Z} / 2-$ action. See [WWd, 9.4] and [WW2, 4.6].

The product map that we need can now be described as a bi- $\Delta-$ map between bi- $\Delta$-spaces, as follows. The Waldhausen product construction of $\S 1$, with $\mathcal{A}^{*}(m)$, $\mathcal{B}^{*}(n)$ and $\mathcal{C}^{*}(m, n)$ instead of $\mathcal{A}, \mathcal{B}$ and $\mathcal{C}$, has a straightforward refinement to

$$
(\bullet \bullet) \quad \Omega\left|x w \mathcal{S}_{\bullet} \mathcal{A}^{*}(m)\right| \wedge \Omega\left|x w \mathcal{S}_{\bullet} \mathcal{B}^{*}(n)\right| \longrightarrow \Omega \Omega\left|x w w \mathcal{S}_{\bullet} \mathcal{S}_{\bullet} \mathcal{C}^{*}(m, n)\right| \text {. }
$$

This involves not only $\diamond: \mathcal{A}^{*}(m) \times \mathcal{B}^{*}(n) \rightarrow \mathcal{C}^{*}(m, n)$, but also the intertwiner $\jmath$. In somewhat more detail: An object of degree $s$ in the simplicial category $x w \mathcal{S}_{k} \mathcal{A}^{*}(m)$ is a pair $(C, D)$ of objects in $\mathcal{S}_{k} \mathcal{A}^{*}(m)$, coupled by means of an $s$-simplex $z$ in $C \odot D$ whose component is nondegenerate. See [WWd, $\S 4]$. Given another object of the same degree $s$ in $x w \mathcal{S}_{\ell} \mathcal{B}^{*}(n)$, consisting of a pair $\left(C^{\prime}, D^{\prime}\right)$ of objects in $\mathcal{S}_{\ell} \mathcal{B}^{*}(n)$ and an $s$-simplex $z^{\prime}$ in a nondegenerate component of $C^{\prime} \odot D^{\prime}$, one finds that $C \diamond C^{\prime}$ and $D \diamond D^{\prime}$ are objects in $\mathcal{S}_{k} \mathcal{S}_{\ell} \mathcal{C}^{*}(m, n)$ coupled by means of the $s$-simplex $\jmath\left(z, z^{\prime}\right)$ which belongs to a nondegenerate component of $\left(C \diamond C^{\prime}\right) \odot\left(D \diamond D^{\prime}\right)$. In this way $\diamond$ and $\jmath$ induce

$$
\left|x w \mathcal{S}_{k} \mathcal{A}^{*}(m)\right| \times\left|x w \mathcal{S}_{\ell} \mathcal{B}^{*}(n)\right| \longrightarrow\left|x w w \mathcal{S}_{k} \mathcal{S}_{\ell} \mathrm{C}^{*}(m, n)\right|
$$

for each $m$ and $n$. This in turn induces $(\bullet \bullet)$. The detailed description shows that

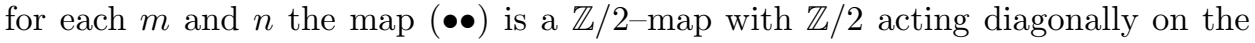
domain (and trivially on all loop coordinates in sight). With the abbreviations

$$
\begin{gathered}
K\left(\mathcal{A}^{*}(m)\right)=\Omega\left|x w \delta . \mathcal{A}^{*}(m)\right|, \\
K\left(\mathcal{B}^{*}(m)\right)=\Omega\left|x w \delta . \mathcal{B}^{*}(m)\right|, \\
K\left(\mathcal{C}^{*}(m, n)\right)=\Omega \Omega\left|x w w \delta . \mathcal{S}_{\bullet} \mathcal{C}^{*}(m, n)\right|
\end{gathered}
$$


we obtain from $(\bullet \bullet)$ a composite map

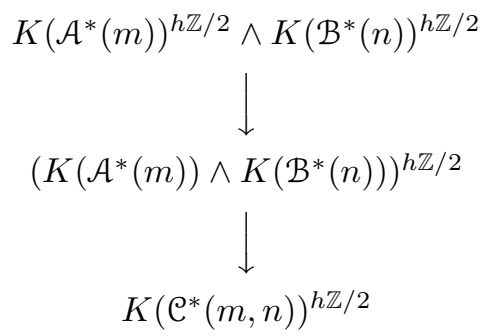

for each $m$ and $n$. On passage to geometric realizations this gives a product map

$$
\Omega^{\infty}\left(\mathbf{K}(\mathcal{A})^{t h \mathbb{Z} / 2}\right) \wedge \Omega^{\infty}\left(\mathbf{K}(\mathcal{B})^{t h \mathbb{Z} / 2}\right) \longrightarrow \Omega^{\infty}\left(\mathbf{K}(\mathcal{C})^{t h \mathbb{Z} / 2}\right) .
$$

\section{Multiplicativity of $\Xi$}

We keep the notation of $\S \S 3$ and 4 . The goal is to establish the commutative diagram in 2.2. One obstruction is that we have not yet given a description of $\Xi: L^{\bullet}(\mathcal{C}) \rightarrow \Omega^{\infty}\left(\mathbf{K}(\mathcal{C})^{t h \mathbb{Z} / 2}\right)$ adapted to the models for $L^{\bullet}(\mathcal{C})$ and $\Omega^{\infty}\left(\mathbf{K}(\mathcal{C})^{t h \mathbb{Z} / 2}\right)$ currently in use. Such a description is easy to supply: an $(m, n)$-bisimplex $(C, \phi)$ in $L^{\bullet}(\mathcal{C})$ determines a homotopy fixed point of the $\mathbb{Z} / 2$-action on

$$
\left|x w \mathcal{C}^{*}(m, n)\right| \cong\left|x w w \mathcal{S}_{1} \mathcal{S}_{1} \mathcal{C}^{*}(m, n)\right| \subset \Omega^{2}\left|x w w \mathcal{S}_{\bullet} \mathcal{S}_{\bullet} \mathcal{C}^{*}(m, n)\right|=: K\left(\mathcal{C}^{*}(m, n)\right),
$$
i.e., a point $\Xi(C, \phi)$ in $K\left(\mathcal{C}^{*}(m, n)\right)^{h \mathbb{Z} / 2}$. The rule $(C, \phi) \mapsto \Xi(C, \phi)$ defines $\Xi$ as a map of bi- $\Delta$-spaces,

$$
\operatorname{sp}_{0}\left(\mathrm{C}^{*}(m, n)\right) \longrightarrow K\left(\mathrm{C}^{*}(m, n)\right)^{h \mathbb{Z} / 2} \quad(m, n \geq 0)
$$

where $\mathrm{sp}_{0}$ denotes the set of 0 -dimensional symmetric Poincaré objects. We must legalize the (re-)definition. To this end note once again that the inclusions of horizontal or vertical 0 -skeletons in the geometric realizations of domain and codomain of $(\bullet)$ are homotopy equivalences. So when it comes to identifying the map we have defined by $(\bullet)$, we can discard the terms with $m>0$. Also, there is a standard inclusion

$$
\Sigma\left|w \mathcal{S}_{\bullet} \mathcal{C}^{*}(0, n)\right| \cong \Sigma\left|w w \mathcal{S}_{1} \mathcal{S}_{\bullet} \mathcal{C}^{*}(0, n)\right| \hookrightarrow\left|w w \mathcal{S}_{\bullet} \mathcal{S}_{\bullet} \mathcal{C}^{*}(0, n)\right|
$$

which leads to a homotopy equivalence $e_{n}: \Omega\left|w \mathcal{S}_{\bullet} \mathcal{C}^{*}(0, n)\right| \rightarrow \Omega \Omega\left|w w \mathcal{S}_{\bullet} \mathcal{S}_{\bullet} \mathcal{C}^{*}(0, n)\right|$. We see that $(\bullet)$ specialized to $m=0$ differs from the standard definition of $\Xi$ only by composition with the degreewise homotopy equivalence $\left\{e_{n} \mid n \geq 0\right\}$. This completes the legalization. With the definitions of $\Xi$ that we now have (the standard one for $\mathcal{A}$ and $\mathcal{B}$, and an inflated one for $\mathcal{C}$ ), commutativity does indeed hold in

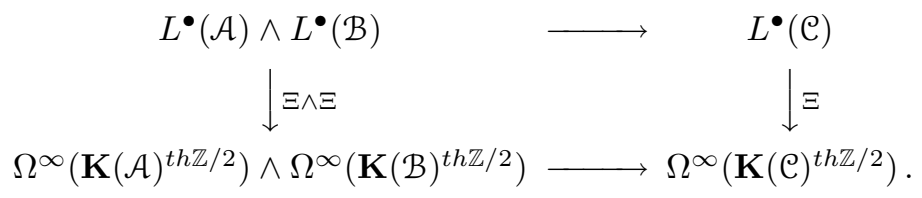

\section{Stable Products}

In this section the goal is to enhance the product maps defined in $\S \S 3,4$ to spectrum level maps and to show that $\Xi$ is multiplicative at the spectrum level. It was stated in 2.2 that this would require more input; what the extra input amounts to, in the notation of 2.2 and 2.3, is more stability in the SW products on $\mathcal{A}, \mathcal{B}, \mathcal{C}$ and in the intertwiner $\mathcal{~}$. It was shown in [WWd, 10.4] that any pointed 
SW product $\odot_{0}$ on a Waldhausen category $\mathcal{A}$ admits a delooping, provided $\mathcal{A}$ has a cylinder functor. In other words, there exists another pointed SW product $\odot_{1}$ on $\mathcal{A}$ and a natural pointed homotopy equivalence from $C \odot_{0} D$ to $\Omega\left(C \odot_{1} D\right)$. The pair $\left(\mathcal{A}, \odot_{0}\right)$ satisfies the axioms $\left.\left.\mathrm{i}\right)-\mathrm{v}\right)$ listed in $\S 2$ if and only if the pair $\left(\mathcal{A}, \odot_{1}\right)$ satisfies them. As a consequence a pointed $\mathrm{SW}$ product $\odot_{0}$ on $\mathcal{A}$ determines a stable SW product $\odot_{\bullet}$. This is a sequence of pointed SW products $\ldots, \odot_{-1}, \odot_{0}, \odot_{1}, \ldots$, together with natural pointed maps $C \odot_{i} D \rightarrow \Omega\left(C \odot_{i+1} D\right)$ which are homotopy equivalences and respect the symmetry $\tau$. The stable SW product can be used, if $\mathcal{A}$ and $\odot$ satisfy axioms i) $-\mathrm{v}$ ) in $\S 2$, to produce a map of $\Omega$-spectra

$$
\Xi: \mathbf{L} \cdot(\mathcal{A}) \rightarrow \mathbf{K}(\mathcal{A})^{t h \mathbb{Z} / 2}
$$

specializing in degree 0 to the map of spaces $\Xi: L^{\bullet}(\mathcal{C}) \rightarrow \Omega^{\infty}\left(\mathbf{K}(\mathcal{C})^{t h \mathbb{Z} / 2}\right)$ which we used in the preceding sections.- Below we will also need bistable SW products. A bistable SW product $\odot$.. on a Waldhausen category $\mathcal{C}$ is a bisequence of pointed $\mathrm{SW}$ products $\odot_{i, j}$ on $\mathcal{C}$ together with natural pointed maps

$$
\begin{aligned}
C \odot_{i, j} D & \rightarrow \Omega\left(C \odot_{i, j+1} D\right), \\
C \odot_{i, j} D & \rightarrow \Omega\left(C \odot_{i+1, j} D\right)
\end{aligned}
$$

which make each collection $C \odot . . D:=\left\{C \odot_{i, j} D\right\}$ into an $\Omega$-bispectrum and respect the symmetry $\tau$.

6.1. Hypotheses. As in the preceding sections we work with three Waldhausen categories $\mathcal{A}, \mathcal{B}$ and $\mathcal{C}$ related by a bi-exact functor $(A, B) \mapsto A \diamond B$ from $\mathcal{A} \times \mathcal{B}$ to $\mathcal{C}$. In addition we assume that $\mathcal{A}, \mathcal{B}$ are equipped with stable $\mathrm{SW}$ products $\odot$. On $\mathcal{C}$, we need a bistable SW product $\odot$... Finally we ask for a natural map of bispectra, the intertwiner:

$$
\jmath:\left(A_{1} \odot{ }_{\bullet} A_{2}\right) \wedge\left(B_{1} \odot \bullet_{2}\right) \rightarrow\left(A_{1} \diamond B_{1}\right) \odot_{\bullet}\left(A_{2} \diamond B_{2}\right)
$$

for $A_{1}, A_{2}$ in $\mathcal{A}$ and $B_{1}, B_{2}$ in $\mathcal{B}$. These data are subject to three conditions. The first is that axioms i $-\mathrm{v})$ above must hold for $\left(\mathcal{A}, \odot_{0}\right)$ and $\left(\mathcal{B}, \odot_{0}\right)$ and $\left(\mathcal{C}, \odot_{0,0}\right)$. The second condition is that the diagram of bispectra

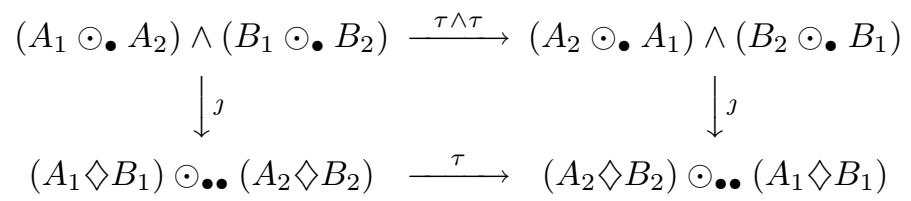

must commute for $A_{1}, A_{2}$ in $\mathcal{A}$ and $B_{1}, B_{2}$ in $\mathcal{B}$. Third condition: If $[\eta] \in$ $\pi_{0}\left(A_{1} \odot_{0} A_{2}\right)$ and $[\zeta] \in \pi_{0}\left(B_{1} \odot_{0} B_{2}\right)$ are nondegenerate [WWd, 3.6], then $[\jmath(\eta \wedge \zeta)]$ is nondegenerate in $\pi_{0}\left(\left(A_{1} \diamond B_{1}\right) \odot_{0,0}\left(A_{2} \diamond B_{2}\right)\right)$.

Remark. It is clear from [WWd] that in the circumstances of 2.3 the pointed SW products on $\mathcal{A}$ and $\mathcal{B}$ can be made stable, and the pointed SW product on $\mathcal{C}$ can be made bistable. Whether it is also possible to improve the intertwiner $\jmath$ to a map of bispectra is another matter. We do not know. It is therefore possible that hypotheses 6.1 are more restrictive than hypotheses 2.3 . Fortunately they are not so restrictive that any of the examples in $\S 2$.A have to be excluded. See $\S 6$.A.

Remark. There is a mild variation on 6.1 where $C \odot_{i} D$ is only defined for $i \geq 0$ and $C, D$ both in $\mathcal{A}$ or both in $\mathcal{B}$, and $C \odot_{i, j} D$ is only defined for $i, j \geq 0$ and $C, D$ in $\mathcal{C}$. This would be sufficient for our purposes. 
In the following $L\left(\mathcal{A}, \odot_{i}\right)$ for example means: $L(\mathcal{A})$ constructed using the $\mathrm{SW}$ product $\odot_{i}$ on $\mathcal{A}$. Also $\mathbf{K}\left(\mathcal{A}, \odot_{i}\right)$ means: $\mathbf{K}(\mathcal{A})$ with the involution determined by the SW product $\odot_{i}$. More details will be supplied as needed.

According to [WWd, 11.1] the spaces $L\left(\mathcal{A}, \odot_{i}\right)$ for $i \in \mathbb{Z}$ form an $\Omega$-spectrum (the structure maps are from $\Sigma L\left(\mathcal{A} \odot_{i}\right)$ to $L\left(\mathcal{A}, \odot_{i+1}\right)$ and their adjoints are homotopy equivalences). Also, the spaces $\Omega^{\infty}\left(\mathbf{K}\left(\mathcal{A}, \odot_{i}\right)^{t h \mathbb{Z} / 2}\right)$ for $i \in \mathbb{Z}$ form an $\Omega$-spectrum. Here $\Omega^{\infty}\left(\mathbf{K}\left(\mathcal{A}, \odot_{i}\right)^{t h \mathbb{Z} / 2}\right)$ is really short for the geometric realization of the $\Delta$-space

$$
m \mapsto K\left(\mathcal{A}, \odot_{i}\right)^{h \mathbb{Z} / 2}
$$

where $K\left(\mathcal{A}, \odot_{i}\right)$ in turn is short for $\Omega\left|x w \mathcal{S}_{.} \mathcal{A}\right|$, where the $x$ prefix has a meaning which depends on the SW product $\odot_{i}$. The maps

$$
\Xi: L\left(\mathcal{A}, \odot_{i}\right) \rightarrow \Omega^{\infty}\left(\mathbf{K}\left(\mathcal{A}, \odot_{i}\right)^{t h \mathbb{Z} / 2}\right)
$$

commute with the structure maps and define therefore a map of $\Omega$-spectra, denoted by the same letter:

$$
\Xi: \mathbf{L}(\mathcal{A}) \longrightarrow \mathbf{K}\left(\mathcal{A}, \odot_{i}\right)^{t h \mathbb{Z} / 2} .
$$

Of course, all this is equally true with $\mathcal{A}$ replaced by $\mathcal{B}$. In fact, by the same mechanism, something similar is true for $\mathrm{C}$ : the maps

$$
\Xi: L\left(\mathcal{C}, \odot_{i, j}\right) \rightarrow \Omega^{\infty}\left(\mathbf{K}\left(\mathcal{C}, \odot_{i, j}\right)^{t h \mathbb{Z} / 2}\right)
$$

commute with the structure maps and define therefore a map of $\Omega$-bispectra, denoted by the same letter:

$$
\Xi: \mathbf{L}(\mathcal{C}) \longrightarrow \mathbf{K}\left(\mathcal{C}, \odot_{i}\right)^{t h \mathbb{Z} / 2} .
$$

Also by inspection, the product maps

$$
\begin{gathered}
L\left(\mathcal{A}, \odot_{i}\right) \wedge L\left(\mathcal{B}, \odot_{j}\right) \rightarrow L\left(\mathcal{C}, \odot_{i, j}\right), \\
\Omega^{\infty}\left(\mathbf{K}\left(\mathcal{A}, \odot_{i}\right)^{t h \mathbb{Z} / 2}\right) \wedge \Omega^{\infty}\left(\mathbf{K}\left(\mathcal{B}, \odot_{j}\right)^{t h \mathbb{Z} / 2}\right) \rightarrow \Omega^{\infty}\left(\mathbf{K}\left(\mathcal{C}, \odot_{i, j}\right)^{t h \mathbb{Z} / 2}\right)
\end{gathered}
$$

induced by $\diamond$ and the bidegree $(i, j)$ part of the intertwiner $\jmath$ commute with the structure maps, and so define maps of bispectra:

$$
\begin{gathered}
\mathbf{L}\left(\mathcal{A}, \odot_{i}\right) \wedge \mathbf{L}\left(\mathcal{B}, \odot_{j}\right) \rightarrow \mathbf{L}\left(\mathcal{C}, \odot_{i, j}\right), \\
\mathbf{K}\left(\mathcal{A}, \odot_{i}\right)^{t h \mathbb{Z} / 2} \wedge \mathbf{K}\left(\mathcal{B}, \odot_{j}\right)^{t h \mathbb{Z} / 2} \rightarrow \mathbf{K}\left(\mathcal{C}, \odot_{i, j}\right)^{t h \mathbb{Z} / 2} .
\end{gathered}
$$

With these specific models, commutativity holds in the diagram of bispectra

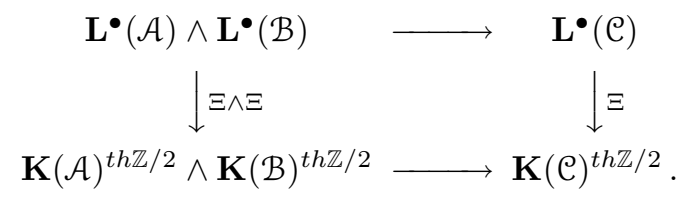

\section{A. Examples}

6.A.1. Example. (Compare 2.A.2.) Given rings with involution $R$ and $S$, let $\mathcal{A}, \mathcal{B}$ and $\mathcal{C}$ be the categories of bounded chain complexes of f.g. projective left 
modules over $R, S$ and $T=R \otimes S$, respectively. Define stable SW products on $\mathcal{A}$ and $\mathcal{B}$, and a bistable $\mathrm{SW}$ product on $\mathcal{C}$, by

$$
\begin{gathered}
C \odot_{i} D:=\left(\Sigma^{i}\left(C \otimes_{R} D\right)\right)^{\sharp}, \\
C \odot_{i} D:=\left(\Sigma^{i}\left(C \otimes_{S} D\right)\right)^{\sharp}, \\
C \odot_{i, j} D:=\left(\Sigma^{i+j}\left(C \otimes_{T} D\right)\right)^{\sharp \sharp},
\end{gathered}
$$

respectively. The structure maps $\Sigma\left(C \odot_{i} D\right) \rightarrow C \odot_{i+1} D$ for $C, D$ both in $\mathcal{A}$ or both in $\mathcal{B}$ are special cases of a natural map of simplicial sets $\Sigma\left(E^{\sharp}\right) \rightarrow(\Sigma E)^{\sharp}$ defined for every chain complex $E$ of abelian groups, as follows. To an $(n-1)$-chain in $E^{\sharp}$, given by a chain map $f$ from the cellular chain complex $\operatorname{cl}\left(\Delta^{n-1}\right)$ to $E$, we associate the composition $\Sigma f \cdot p$ from $\operatorname{cl}\left(\Delta^{n}\right)$ to $\Sigma E$. Here $p: \operatorname{cl}\left(\Delta^{n}\right) \rightarrow \Sigma \operatorname{cl}\left(\Delta^{n-1}\right)$ can be defined as the map of reduced cellular chain complexes induced by a canonical quotient map of CW-spaces, $\Delta_{+}^{n} \rightarrow \Sigma\left(\Delta_{+}^{n-1}\right)$. The structure maps in $C \odot . . D$ for $C, D$ in $\mathcal{C}$ are defined similarly ; they are special cases of natural bisimplicial maps $\Sigma_{h}\left(E^{\sharp \sharp}\right) \rightarrow(\Sigma E)^{\sharp \sharp}$ and $\Sigma_{v}\left(E^{\sharp \sharp}\right) \rightarrow(\Sigma E)^{\sharp \sharp}$ where $\Sigma_{h}$ and $\Sigma_{v}$ denote horizontal and vertical suspension respectively (certain functors from pointed bisimplicial sets to pointed bisimplicial sets). Finally the construction of $\jmath$ as a map of bispectra from $\left(A_{1} \odot A_{2}\right) \wedge\left(B_{1} \odot B_{2}\right)$ to $\left(A_{1} \diamond B_{1}\right) \odot \bullet .\left(A_{2} \diamond B_{2}\right)$ is completely analogous to what we had in 2.A.3. To avoid sign troubles, avoid using the symbol $\Sigma$; use instead $\mathbb{S}^{1} \wedge$ or $(\mathbb{Z}, i) \otimes$ as appropriate, where $(\mathbb{Z}, i)$ is the chain complex of free abelian groups equal to $\mathbb{Z}$ in degree $i$ and zero elsewhere.

6.A.2. Example. Compare 2.A.3. Let $\mathcal{A}, \mathcal{B}, \mathcal{C}$ and $\diamond$ be as in 2.A.3. For $\mathbf{X}$ and $\mathbf{Y}$ both in $\mathcal{A}=\mathcal{B}$ and $i \geq 0$ let

$$
\mathbf{X} \odot_{i} \mathbf{Y}:=\Omega^{\infty}\left(\mathbb{S}^{i} \wedge \mathbf{X} \wedge \mathbf{Y}\right),
$$

or more precisely: the simplicial set whose $n$-simplices are the bispectrum maps from $\Delta_{+}^{n} \wedge \mathbf{S}^{0} \wedge \mathbf{S}^{0}$ to $\mathbb{S}^{i} \wedge \mathbf{X} \wedge \mathbf{Y}$. For $\mathbf{X}, \mathbf{Y}$ in $\mathcal{C}$ and $i, j \geq 0$ let

$$
\mathbf{X} \odot_{i, j} \mathbf{Y}:=\Omega^{\infty}\left(\mathbb{S}^{i} \wedge \mathbb{S}^{j} \wedge \mathbf{X} \wedge \mathbf{Y}\right),
$$

more precisely: the bisimplicial set whose $(m, n)$-simplices are the 4 -spectrum maps from $\Delta_{+}^{m} \wedge \Delta_{+}^{n} \wedge \mathbf{S}^{0} \wedge \mathbf{S}^{0} \wedge \mathbf{S}^{0} \wedge \mathbf{S}^{0}$ to $\mathbb{S}^{i} \wedge \mathbb{S}^{j} \wedge \mathbf{X} \wedge \mathbf{Y}$. The intertwiner $\jmath$ is defined (modulo obvious identifications, as a map of bispectra made from bisimplicial sets) by $(f, g) \mapsto f \wedge g$ for an $m$-simplex $f \in \mathbf{X} \odot \mathbf{Y}$ and an $n$-simplex $g \in \mathbf{X}^{\prime} \odot \mathbf{Y}^{\prime}$, with $\mathbf{X}, \mathbf{Y}$ in $\mathcal{A}$ and $\mathbf{X}^{\prime}, \mathbf{Y}^{\prime}$ in $\mathcal{B}$. The definition of $\tau$ is obvious in the case of $\mathcal{A}$ and $\mathcal{B}$, and it is analogous to that in 6.A.1 in the case of $\mathcal{C}$.

\section{Associativity}

Our results so far include the statement that $\Xi: \mathbf{L}(\mathcal{C}) \rightarrow \mathbf{K}(\mathcal{C})^{t h \mathbb{Z} / 2}$ is a multiplicative map of spectra if $\mathcal{C}$ is the category of bounded chain complexes of finitely generated free abelian groups (for example). But the proof involves two distinct models for each of the spectra $\mathbf{L}(\mathcal{C}), \mathbf{K}(\mathcal{C})$, making it nontrivial to state and prove that $\Xi$ is a map of ring spectra. However, with a definition of ring spectrum adapted to the purpose ( 7.1 below), it can be done.

In definition 7.1, we mean by an $n$-spectrum a collection of pointed CW-spaces $X_{\alpha}$ for $\alpha \in \mathbb{Z}^{n}$, together with structure maps $\Sigma X_{\alpha} \rightarrow X_{\beta}$ which are, up to CWisomorphism, inclusions of CW-subspaces and are defined whenever $\beta-\alpha$ has a single nonzero coordinate, and that is equal to 1 . The structure maps are subject to the usual relations. For instance, a 1 -spectrum is an ordinary $\mathrm{CW}$-spectrum, a 
2-spectrum is a CW-bispectrum, and so on. We make n-spectra into a category following Boardman [Ad1, Part III]: a morphism is an equivalence class of functions. We insist on cellular maps for simplicity.

7.1. Definition. A ring multispectrum consists of

1. an $n$-spectrum $\mathbf{F}(n)$ for each $n>0$;

2. maps of $(m+n)$-spectra $\mu: \mathbf{F}(m) \wedge \mathbf{F}(n) \rightarrow \mathbf{F}(m+n)$ (for all $m, n>0$ ) which are associative ;

3. a map of 1-spectra $\eta: \mathbf{S}^{0} \longrightarrow \mathbf{F}(1)$ such that the following compositions are homotopy equivalences for every $n>0$ :

$$
\begin{aligned}
& \mathbf{S}^{0} \wedge \mathbf{F}(n) \stackrel{\eta \wedge \mathrm{id}}{\longrightarrow} \mathbf{F}(1) \wedge \mathbf{F}(n) \stackrel{\mu}{\longrightarrow} \mathbf{F}(1+n), \\
& \mathbf{F}(n) \wedge \mathbf{S}^{0} \stackrel{\text { id } \wedge \eta}{\longrightarrow} \mathbf{F}(n) \wedge \mathbf{F}(1) \stackrel{\mu}{\longrightarrow} \mathbf{F}(n+1) .
\end{aligned}
$$

Remark. The underlying spectrum $\mathbf{F}(1)$ of a ring multispectrum $\mathbf{F}(?)$ as in 7.1 has a homotopy associative multiplication with two-sided homotopy unit.

Proof/Explanation. Let $\mathcal{H}(n)$ be the homotopy category of $n$-spectra. The internal smash product $\mathcal{H}(1) \times \mathcal{H}(1) \rightarrow \mathcal{H}(1)$ that we use here will be denoted by $\curlywedge$, to avoid confusion with the obvious smash product $\wedge: \mathcal{H}(1) \times \mathcal{H}(1) \rightarrow \mathcal{H}(2)$. It is defined implicitly by a natural bijection

$$
[\mathbf{X} \wedge \mathbf{Y}, \mathbf{W}] \cong\left[\mathbf{X} \wedge \mathbf{Y}, \mathbf{S}^{0} \wedge \mathbf{W}\right]
$$

for $\mathbf{W}$ in $\mathcal{H}(1)$, where the square brackets denote morphism sets in $\mathcal{H}(1)$ and $\mathcal{H}(2)$, respectively. The implicit definition is justified because $\mathbf{S}^{0} \wedge$ is an equivalence $\mathcal{H}(1) \rightarrow \mathcal{H}(2)$. Now let $\mathbf{F}($ ?) be a ring multispectrum. Then the composition of $\mu: \mathbf{F}(1) \wedge \mathbf{F}(1) \rightarrow \mathbf{F}(2)$ with the inverse of $\mu(\eta \wedge$ id $): \mathbf{S}^{0} \wedge \mathbf{F}(1) \rightarrow \mathbf{F}(2)$ is an element in $\left[\mathbf{F}(1) \wedge \mathbf{F}(1), \mathbf{S}^{0} \wedge \mathbf{F}(1)\right] \cong[\mathbf{F}(1) \curlywedge \mathbf{F}(1), \mathbf{F}(1)]$ which we can view as a multiplication $\mathbf{F}(1) \curlywedge \mathbf{F}(1) \rightarrow \mathbf{F}(1)$. From the conditions in 7.1, the multiplication is associative and has $[\eta] \in \pi_{0}(\mathbf{F}(1))$ as a left unit. On the right, $[\eta]$ acts invertibly. But $[\eta]^{2}=[\eta]$ since $[\eta]$ is a left unit, so $[\eta]$ must be a right unit, too.

7.2. Example. Let $\mathcal{C}$ be the category of bounded chain complexes of finitely generated free abelian groups, with the stable SW product $\odot$. specified in 6.A.1, that is,

$$
C \odot_{i} D:=\left(\Sigma^{i}(C \otimes D)\right)^{\sharp} .
$$

Then $\mathbf{L} \bullet(\mathcal{C})$ is the underlying spectrum $\mathbf{E}(1)$ of a ring multispectrum $\mathbf{E}($ ?).

Proof, in outline. In more detail, $\mathbf{E}(1)$ is the 1 -spectrum whose $i$-th term is the $\Delta$-set $L^{\bullet}\left(\mathcal{C}, \odot_{i}\right)$, so that the $m$-simplices in the $i$-th term are the 0 -dimensional symmetric Poincaré objects in $\mathcal{C}^{*}(m)$ with respect to the $\mathrm{SW}$ product $\odot_{i}$. We have seen $\mathbf{E}(2)$ constructed in $\S 6$ and $\S 6$.A. The input is a bistable SW product $\odot$.. on $\mathcal{C}$, defined as in 6. A.1 by the formula

$$
C \odot_{i j} D:=\left(\Sigma^{i+j}(C \otimes D)\right)^{\sharp} .
$$

The $(i, j)$-term of $\mathbf{F}(2)$ is defined as the bi- $\Delta$-set whose $(m, n)$-bisimplices are the 0 -dimensional symmetric Poincaré objects in $\mathcal{C}^{*}(m, n)$ with respect to the $\mathrm{SW}$ product $\odot_{i j}$. The multiplication $\mu: \mathbf{E}(1) \wedge \mathbf{E}(1) \rightarrow \mathbf{E}(2)$ has been described in $\S 6$ and 6.A.1. In order to construct $\mathbf{E}(k)$ for $k>2$, we need first a $k$-stable $\mathrm{SW}$ product on $\mathcal{C}$ (a functor from $\mathcal{C} \times \mathcal{C}$ to $k$-spectra). The definition is an obvious generalization of 6.A.1. Then we define the $\left(i_{1}, \ldots, i_{k}\right)$-term of $\mathbf{E}(k)$ as the $k-\Delta$-set 
whose $\left(m_{1}, \ldots, m_{k}\right)$-simplices are the 0-dimensional symmetric Poincaré objects in $\mathcal{C}^{*}\left(m_{1}, \ldots, m_{k}\right)$ with respect to the ordinary SW product which is the $\left(i_{1}, \ldots, i_{k}\right)-$ term in the given $k$-stable SW product. Multiplication $\mu$ and unit $\eta$ are obvious. To ensure that $\mu$ is associative, use a definition of tensor product (of finitely generated free abelian groups) which is strictly associative, not just associative up to coherent isomorphism. This can be arranged. To check that $\eta$ satisfies the conditions in 7.1, look at homotopy groups, noting that all (multi-) $\Delta$-sets in sight are fibrant.

7.3. Example. Keep $\mathcal{C}$ and the stable SW product $\odot$. from 7.2 . Then $\mathbf{K}(\mathcal{C})^{t h \mathbb{Z} / 2}$ is the underlying spectrum of a ring multispectrum $\mathbf{F}(?)$. Idea of proof: exactly as in 7.2 .

7.4. Example. Let $\mathcal{C}$ be the Waldhausen category of CW-spectra with finitely many cells, with the stable SW product specified in 6.A.2:

$$
\mathbf{X} \diamond \mathbf{Y}:=\Omega^{\infty}(\mathbf{X} \wedge \mathbf{Y})
$$

Then $\mathbf{L}^{\bullet}(\mathcal{C})$ and $\mathbf{K}(\mathcal{C})^{t h \mathbb{Z} / 2}$ are the underlying spectra of ring multispectra $\mathbf{E}(?)$ and $\mathbf{F}(?)$, respectively.

7.5. Conclusion. With $\mathcal{C}$ as in 7.2 and 7.3 or as in 7.4 , the map $\Xi: \mathbf{L} \bullet \mathcal{C}) \rightarrow$ $\mathbf{K}(\mathcal{C})^{t h \mathbb{Z} / 2}$ can be refined to a map of ring multispectra.

Proof. The appropriate ring multispectra have just been described, and $\Xi$ extends for obvious reasons (compare $\S 5, \S 6$ ).

7.6. Proposition. With $\mathcal{C}$ as in 7.2 and 7.3 or 7.4 , the unit $1 \in \pi_{0}\left(\mathbf{K}(\mathbb{Z})^{\text {th } \mathbb{Z} / 2}\right)$ has infinite order.

Preliminaries for the proof. Our argument involves a calculation in real topological $K$-theory. It is possible that the connective real topological $K$-theory spectrum bo has a description in terms of chain complexes, analogous to that we have been using for the algebraic $K$-theory of $\mathbb{Z}$, but such a description has not been worked out as far as we know. This forces us to use older models for $K$-theory spectra. Let $R$ be any ring. According to Segal [Seg], the symmetric monoidal category $\mathcal{M}(R)$ of finitely generated projective left $R$-modules, with isomorphisms as morphisms, determines a (special) $\Gamma$-space with underlying space $|\mathcal{M}(R)|$, and therefore a connective spectrum. We may call this the algebraic $K$-theory spectrum of $R$. If the ring $R$ comes with an involution (involutory anti-automorphism), then it is a good idea to enlarge $\mathcal{N}(R)$ a little, as follows. Let $x \mathcal{M}(R)$ have objects $(P, Q, z)$ where $P, Q$ are in $\mathcal{M}(R)$ and $z: P^{*} \rightarrow Q$ is an isomorphism of left $R$-modules. (Here $P^{*}$ is $\operatorname{hom}_{R}(P, R)$, made into a left $R$-module using the involution on $R$.) A morphism $(P, Q, z) \rightarrow\left(P^{\prime}, Q^{\prime}, z^{\prime}\right)$ in $x \mathcal{M}(R)$ is a pair of isomorphisms $i: P \rightarrow P^{\prime}$ and $j: Q \rightarrow Q^{\prime}$ such that $j z i^{*}=z^{\prime}$. (If we identify $Q$ with the dual of $P$ and $Q^{\prime}$ with the dual of $P^{\prime}$ using $z$ and $z^{\prime}$ respectively, this means that $j$ is the inverse of the transpose of $i$.) Clearly $x \mathcal{M}(R)$ is still a symmetric monoidal category, equivalent to $\mathcal{M}(R)$ as such. But it comes with an involution $(P, Q, z) \mapsto(Q, P, \bar{z})$ where $\bar{z}: Q^{*} \rightarrow P$ is the adjoint of $z$. This then leads to an involution on the algebraic $K$-theory spectrum $\mathbf{K}(R)$, provided we define $\mathbf{K}(R)$ using the symmetric monoidal category $x \mathcal{M}(R)$ and Segal's $\Gamma$-space machinery.

The Segal type model of $\mathbf{K}(R)$, made from $x \mathcal{M}(R)$, is contained in the larger model of $\mathbf{K}(R)$ that we normally use, the one defined in terms of the category of bounded chain complexes of f.g. projective left modules over $R$, and an SW 
product as in 2.A.2. The inclusion is a homotopy equivalence, and respects the involutions.

Proof of 7.6. It is sufficient to consider the case where $\mathcal{C}$ is the category of 7.2 , so that $\mathbf{K}(\mathcal{C}) \simeq \mathbf{K}(\mathbb{Z})$. Let bo be the connective $K$-theory spectrum. The standard map from $\mathbf{K}(\mathbb{Z})$ to bo (via the algebraic $K$-theory spectrum of $\mathbb{R}$ ) can be made into a map of spectra with $\mathbb{Z} / 2$-action, but to see this we have to use the Segal model for $\mathbf{K}(\mathbb{Z})$. That is, we think of $\mathbf{K}(\mathbb{Z})$ as the spectrum obtained from the symmetric monoidal category with involution $x \mathcal{M}(\mathbb{Z})$. We think of bo as the spectrum obtained by Segal's method from a topological symmetric monoidal category with involution $x \mathcal{M}\left(\mathbb{R}_{t}\right)$ whose objects are triples $(V, W, z)$ where $V$ and $W$ are finite dimensional real vector spaces and $z: V^{*} \rightarrow W$ is a linear isomorphism. This establishes the claim that the standard map from $\mathbf{K}(\mathcal{C}) \simeq \mathbf{K}(\mathbb{Z})$ to bo can be viewed as a map of spectra with involution, and it also shows that the appropriate involution on bo is essentially trivial. Namely, $x \mathcal{M}\left(\mathbb{R}_{t}\right)$ has a full subcategory, equivalent to the whole, consisting of the objects $\left(\mathbb{R}^{m}, \mathbb{R}^{m}, z\right)$ where $z$ is the standard isomorphism from the dual of $\mathbb{R}^{m}$ to $\mathbb{R}^{m}$. On this subcategory, the involution is actually trivial. We finish the argument as follows. The norm fibration sequences for $\mathbf{K}(\mathbb{Z})$ and bo give us a commutative diagram with exact rows

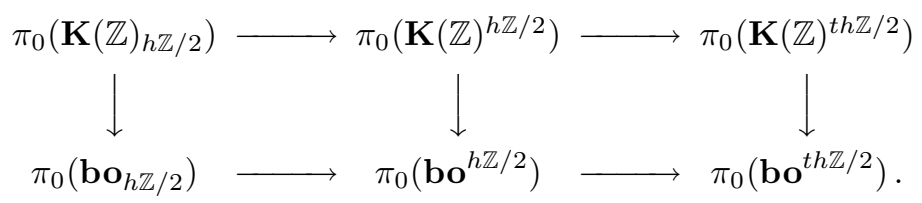

It is enough to show that the image of $1 \in \pi_{0}\left(\mathbf{K}(\mathbb{Z})^{t h \mathbb{Z} / 2}\right)$ in $\pi_{0}\left(\mathbf{b o}^{t h \mathbb{Z} / 2}\right)$ has infinite order. This image has a canonical lift to $\pi_{0}\left(\mathbf{b o}{ }^{h \mathbb{Z} / 2}\right)$, represented by the trivial line bundle on $\mathbb{R} P^{\infty}$. By the calculations of [AtSe], the trivial line bundle on $\mathbb{R} P^{\infty}$ represents an element of infinite order in the cokernel of the homomorphism $\pi_{0}\left(\mathbf{b o}_{h \mathbb{Z} / 2}\right) \rightarrow \pi_{0}\left(\mathbf{b o}^{h \mathbb{Z} / 2}\right)$.

In more detail: $\pi_{0}\left(\right.$ boo $\left.^{h \mathbb{Z} / 2}\right)$ is isomorphic to $\mathbb{Z} \oplus \mathbb{Z}_{2}^{\wedge}$ and the standard generators for the two summands are $[\varepsilon]$ and $[\varepsilon]-[\eta]$, where $\varepsilon$ is the trivial line bundle on $\mathbb{R} P^{\infty}$ and $\eta$ is the nontrivial line bundle on $\mathbb{R} P^{\infty}$. (These "generators" only generate a dense subgroup of $\pi_{0}$ (bo ${ }^{h \mathbb{Z} / 2}$ ), to be precise.) The inclusion bo $\rightarrow \mathbf{b o}_{h \mathbb{Z} / 2}$ induces an isomorphism in $\pi_{0}$ so that $\pi_{0}\left(\mathbf{b o}_{h \mathbb{Z} / 2}\right) \cong \mathbb{Z}$. The norm homomorphism sends the standard generator of $\pi_{0}\left(\mathbf{b o}_{h \mathbb{Z} / 2}\right)$ to the bundle on $\mathbb{R} P^{\infty}$ manufactured from the permutation representation of $\mathbb{Z} / 2$ on $\mathbb{R}^{2}$; this is in the class $[\varepsilon]+[\eta]$.

7.7. Corollary. The homomorphism $\Xi_{*}$ from $\pi_{4 k}(\mathbf{L} \bullet(\mathbb{Z})) \cong \mathbb{Z}$ to $\pi_{4 k}\left(\mathbf{K}(\mathbb{Z})^{\text {th } \mathbb{Z} / 2}\right)$ is injective, for any $k \in \mathbb{Z}$.

Proof. The graded homomorphism $\Xi_{*}$ from $\pi_{*}\left(\mathbf{L}^{\bullet}(\mathbb{Z})\right)$ to $\pi_{*}\left(\mathbf{K}(\mathbb{Z})^{t h \mathbb{Z} / 2}\right)$ is 4 -periodic by 7.5 , since its domain has an invertible element in degree 4 . By 7.6, it is also injective in degree 0.

7.8. Definition. A module multispectrum over a ring multispectrum $\mathbf{F}($ ?) $=$ $(\mathbf{F}(?), \mu, \eta)$ consists of

1. an $n$-spectrum $\mathbf{G}(n)$ for each $n>0$;

2. maps of $(m+n)$-spectra $\rho: \mathbf{F}(m) \wedge \mathbf{G}(n) \rightarrow \mathbf{G}(m+n)$ (for all $m, n>0$ ) which are subject to two conditions. The first is associativity: the two evident maps from $\mathbf{F}(\ell) \wedge \mathbf{F}(m) \wedge \mathbf{G}(n)$ to $\mathbf{G}(\ell+m+n)$ defined in terms of $\rho$ and $\mu$ agree, for 
arbitrary $\ell, m, n>0$. The second concerns the unit. Namely, the composition of $\rho: \mathbf{F}(1) \wedge \mathbf{G}(n) \rightarrow \mathbf{G}(1+n)$ with $\eta \wedge$ id $: \mathbf{S}^{0} \wedge \mathbf{G}(n) \rightarrow \mathbf{F}(1) \wedge \mathbf{G}(n)$ is a homotopy equivalence $\mathbf{S}^{0} \wedge \mathbf{G}(n) \simeq \mathbf{G}(1+n)$.

Remark. Keep the notation of 7.8. In the homotopy category of 1-spectra, $\mathbf{G}(1)$ is a module over $\mathbf{F}(1)$.

7.9. Example. For any ring with involution $R$, the spectrum $\mathbf{K}(R)^{t h \mathbb{Z} / 2}$ is the 1-term of a module multispectrum over a ring multispectrum whose 1-term is $\mathbf{K}(\mathbb{Z})^{t h \mathbb{Z} / 2}$. (Here $\mathbf{K}(R)$ is short for $\mathbf{K}(\mathcal{B})$ where $\mathcal{B}$ is the category of bounded chain complexes of f.g. projective $R$-modules.)

7.10. Corollary. The spectrum $\mathbf{K}(R)^{t h \mathbb{Z} / 2}$ is a generalized Eilenberg-Mac Lane spectrum, for every (discrete) ring with involution $R$. It is also $4-$ periodic:

$$
\mathbf{K}(R)^{t h \mathbb{Z} / 2} \simeq \Omega^{4} \mathbf{K}(R)^{t h \mathbb{Z} / 2} .
$$

Proof. $\mathbf{K}(R)^{t h \mathbb{Z} / 2}$ is a 2-local spectrum, and (in the homotopy category of 1spectra) a module over the ring spectrum $\mathbf{K}(\mathbb{Z})^{t h \mathbb{Z} / 2}$ and then also over $\mathbf{L} \bullet(\mathbb{Z})$. Now $\mathbf{L}^{\bullet}(\mathbb{Z})$ is 4 -periodic with the definition we use, which agrees with [Ran2] but not with [Ran1]. (In other words: there exists an invertible element in $\pi_{4}\left(\mathbf{L}^{\bullet}(\mathbb{Z})\right)$.) Further, $\mathbf{L}^{\bullet}(\mathbb{Z})$ is an algebra over the ring spectrum MSO which is a generalized Eilenberg-Mac Lane spectrum when localized at the prime 2. See [TW].

Remark. Let $\mathcal{C}$ be the Waldhausen category of $\mathrm{CW}$-spectra with finitely many cells, as in 7.4. Then $\mathbf{K}(\mathcal{C})^{t h \mathbb{Z} / 2}$ is a module over $\mathbf{L}^{\bullet}(\mathcal{C})$, but $\mathbf{L}^{\bullet}(\mathcal{C})$ is not 4 -periodic, nor is it an algebra over MSO. We can not conclude that $\mathbf{K}(\mathcal{C})^{t h \mathbb{Z} / 2}=\mathbf{A}(*)^{t h \mathbb{Z} / 2}$ is generalized Eilenberg-Mac Lane or that it is 4 -periodic. It is certainly not generalized Eilenberg-Mac Lane.

In fact it can be shown that Waldhausen's splitting of $\mathbf{K}(\mathcal{C})=\mathbf{A}(*)$ into $\mathbf{S}^{0}$ and another wedge summand is invariant under the duality involution. The resulting involution on $\mathbf{S}^{0}$ is trivial. Therefore $\mathbf{K}(\mathcal{C})^{t h \mathbb{Z} / 2}$ contains the Tate spectrum of $\mathbb{Z} / 2$ acting trivially on $\mathbf{S}^{0}$ as a wedge summand, up to homotopy equivalence. By the affirmed Segal conjecture [Car], [Lin] the Tate spectrum of $\mathbb{Z} / 2$ acting trivially on $\mathbf{S}^{0}$ is homotopy equivalent to the completion at 2 of the sphere spectrum. It is not a wedge summand of a generalized Eilenberg-Mac Lane spectrum.

\section{Applications}

Overview. We will use the results of the previous sections to make partial calculations of homotopy groups of certain spectra $\mathbf{L K} \bullet(\mathcal{C}, n)$ which are obtained by mixing the quadratic $L$-theory and the algebraic $K$-theory of a Waldhausen category $\mathcal{C}$ with SW products. It is explained in [WWaut] how these spectra are related to manifold geometry, in particular to the homotopy theory of $h$-structure spaces $\mathcal{S}(M)$. In this way, our partial calculations lead to results about homotopy groups of such $h$-structure spaces, and related spaces such as spaces of smooth and topological automorphisms of manifolds.

8.1. Definitions. For $n \geq 0$ let $\mathbb{S}_{!}^{n}$ be the one-point compactification of $\mathbb{R}^{n}$, viewed as a space with involution $z \mapsto-z$. The fixed point set of this involution on $\mathbb{S}_{!}^{n}$ is $\mathbb{S}_{!}^{0}=\{0, \infty\}$. For a Waldhausen category $\mathcal{C}$ with $\mathrm{SW}$-duality, let

$$
t^{n}: \mathbf{K}(\mathcal{C})^{t h \mathbb{Z} / 2} \longrightarrow\left(\mathbb{S}_{!}^{n} \wedge \mathbf{K}(\mathcal{C})\right)^{t h \mathbb{Z} / 2}
$$


be the inclusion (which is a homotopy equivalence, by an easy induction on $n$ ). Let LK. $(\mathcal{C}, n)$ be the homotopy pullback of

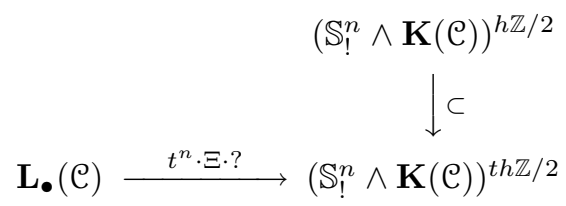

where ? denotes the forgetful map from quadratic to symmetric $L$-theory. (Compare $\S 4.2$ of [WWaut]; beware that we use a 0 -connected version of $\mathbf{L}_{\bullet}(\mathcal{C})$ in [WWaut].)

If $\mathcal{C}$ is the category of chain complexes of f.g. projective left $R$-modules, bounded below and above, with the usual SW product (compare 2.A.2), then we also write LK. $(R, n)$ instead of $\mathbf{L K} \cdot(\mathrm{C})$, and

$$
L K_{k}(R, n):=\pi_{k}\left(\mathbf{L K}_{\bullet}(R, n)\right) \quad(k \in \mathbb{Z}) .
$$

Another special case which calls for special notation is the following. Suppose that $B$ is any space and $\gamma$ is a spherical fibration on $B$, with fibers $\simeq \mathbb{S}^{i}$ and a distinguished section which is a cofibration (and which we call the zero section). Suppose that $\mathcal{C}$ is the category of retractive spaces over $B$ which are equipped with a CW-structure relative to $B$, with finitely many cells only. Then $\mathcal{C}$ is a Waldhausen category, with an SW product determined by $\gamma$ which we will describe just below. In this situation we also write $\mathbf{L A} \bullet(B, n)$ for $\mathbf{L K} \bullet(\mathcal{C}, n)$; and incidentally, we may write $\mathbf{L}_{\bullet}(B)$ for $\mathbf{L}_{\bullet}(\mathcal{C})$, with decorations such as superscripts $s, p$, as appropriate. (As explained in [WWaut, 4.2.3] the mixed theories LA.( $(\ldots, n)$ alias LK. $(\ldots, n)$ are insensitive to decorations as far as homotopy groups $\pi_{k}$ with $k>n$ are concerned, so we don't decorate them as a rule.)

The $\mathrm{SW}$ product on $\mathcal{C}$ is as follows. Suppose that $X, Y$ are objects of $\mathcal{C}$. Identify $\operatorname{holim}(X \rightarrow B \leftarrow Y)$ with the space of triples $(x, \omega, y)$ where $x \in X$ and $y \in Y$, and $\omega:[-1,1] \rightarrow B$ is a path connecting the images of $x$ and $y$ in $B$. We view holim $(X \rightarrow B \leftarrow Y)$ as a retractive space over $B$, with retraction given by $(x, \omega, y) \mapsto \omega(0)$. Then we let

$$
X \odot Y:=\Omega^{\infty+i} \frac{\Sigma^{\gamma} \operatorname{holim}(X \rightarrow B \leftarrow Y)}{\Sigma^{\gamma} B} .
$$

Here we have used $\Sigma^{\gamma}$ as a shorthand notation for Thom spaces; so $\Sigma^{\gamma} B$ is the Thom space of $\gamma$ (total space modulo image of zero section) and $\Sigma^{\gamma}$ holim $(X \rightarrow B \leftarrow Y)$ is the Thom space of the pullback of $\gamma$ to $\operatorname{holim}(X \rightarrow B \leftarrow Y$ ). (Compare 1.A.3 and 1.A.9 of [WWd]; our conventions here are slightly different because we want to ensure that our SW product remains essentially unchanged when we stabilize $\gamma$.) Note in any case that the SW product, and hence LA. $(B, n)$, depend not only on $B$ and $n$, but also on a spherical fibration $\gamma$ (on $B$ ).

8.2. Notation. Our calculations will involve the numbers $a_{q}$ and $a_{q}^{c}$ from the theory of Clifford algebras. Here $a_{q}$ is the dimension of the even part of any irreducible (nontrivial) $\mathbb{Z} / 2$-graded module for the Clifford algebra $\operatorname{Cliff}\left(\mathbb{R}^{q}\right)$, and $a_{q}^{c}$ is the dimension of the even part of any irreducible (nontrivial) $\mathbb{Z} / 2$-graded module for $\mathbb{C} \otimes_{\mathbb{R}} \operatorname{Cliff}\left(\mathbb{R}^{q}\right)$. Explicitly, $a_{q}=1,2,4,4,8,8,8,8$ if $q=1,2,3,4,5,6,7,8$ and $a_{q+8}=16 a_{q}$; further $a_{q}^{c}=1$ if $q=1,2$ and $a_{q+2}^{c}=2 a_{q}^{c}$. See [ABS].

For the calculations, fix a finite group $\pi$, with group algebra $\mathbb{R} \pi$ which we equip with the standard involution. Recall that $L_{k}^{p}(\mathbb{R} \pi)$ can be described in terms of 
"multisignatures". Namely, $L_{k}^{p}(\mathbb{R} \pi)$ splits as a direct sum $\bigoplus L_{k}^{p}\left(E_{V}\right)$ where $V$ runs through a maximal set of pairwise non-isomorphic irreducible real representations of $\pi$, and $E_{V}$ is the endomorphism ring of $V$, isomorphic to $\mathbb{R}, \mathbb{C}$ or $\mathbb{H}$ equipped with a standard involution [Le], [Wa]. It is known that $L_{k}^{p}\left(E_{V}\right) \cong \mathbb{Z}$ if $4 \mid k$, and also if $2 \mid k$ and $E_{V} \cong \mathbb{C}$; otherwise $L_{k}^{p}\left(E_{V}\right)=0$.

8.3. Calculation. The $V$-component of the multisignature of an element in $L_{k}^{p}(\mathbb{R} \pi)$ which lifts to $L K_{k}(\mathbb{R} \pi, n)$, with $k>n$, is divisible by

$$
\begin{array}{lr}
2 a_{k-n} & \left(\text { assuming } 4 \mid k \text { and } E_{V} \cong \mathbb{R}\right), \\
2 a_{k-n}^{c} & \left(\text { assuming } 2 \mid k \text { and } E_{V} \cong \mathbb{C}\right), \\
a_{k-n+4} / 4 & \text { (assuming } \left.4 \mid k \text { and } E_{V} \cong \mathbb{H}\right) .
\end{array}
$$

Proof. It is easy to reduce to the following statement. Let $\mathbb{F}=\mathbb{R}, \mathbb{C}, \mathbb{H}$ and suppose that $k$ is even (divisible by 4 if $\mathbb{F}=\mathbb{R}, \mathbb{H}$ ), and $k>n$. Then the image of $L K_{k}(\mathbb{F}, n)$ in $L_{k}^{p}(\mathbb{F}) \cong \mathbb{Z}$ is contained in the ideal generated by

$$
\begin{array}{lc}
2 a_{k-n} & (\mathbb{F}=\mathbb{R}), \\
2 a_{k-n}^{c} & (\mathbb{F}=\mathbb{C}), \\
a_{k-n+4} / 4 & (\mathbb{F}=\mathbb{H}) .
\end{array}
$$

The proofs in the three cases are similar, so let's concentrate on the case $\mathbb{F}=\mathbb{R}$. Then we may assume $4 \mid k$. We know already from the periodicity results in $\S 7$ (especially proof of 7.6) that the composition

$$
L_{k}^{p}(\mathbb{R}) \stackrel{\Xi}{\longrightarrow} \pi_{k}\left(\mathbf{K}(\mathbb{R})^{t h \mathbb{Z} / 2}\right) \longrightarrow \pi_{k}\left(\mathbf{b o}^{t h \mathbb{Z} / 2}\right)
$$

can be identified with the standard inclusion $\mathbb{Z} \rightarrow \mathbb{Z}_{2}^{\wedge}$. Hence it is enough to show that if an element in

$$
\mathbb{Z}_{2}^{\wedge} \cong \pi_{k}\left(\mathbf{b o}^{t h \mathbb{Z} / 2}\right) \cong \pi_{k}\left(\left(\mathbb{S}_{!}^{n} \wedge \mathbf{b o}\right)^{t h \mathbb{Z} / 2}\right)
$$

lifts to $\pi_{k}\left(\left(\mathbb{S}_{!}^{n} \wedge \mathbf{b o}\right)^{h \mathbb{Z} / 2}\right)$, then it is divisible by $2 a_{k-n}$. Equivalently, it is enough to show that if an element in

$$
\mathbb{Z} \oplus \mathbb{Z}_{2}^{\wedge} \cong \pi_{k}\left(\left(\mathbb{S}_{!}^{k} \wedge \mathbf{b o}\right)^{h \mathbb{Z} / 2}\right)
$$

lifts to $\pi_{k}\left(\left(\mathbb{S}_{!}^{n} \wedge \mathbf{b o}\right)^{h \mathbb{Z} / 2}\right)$, then its component in $\mathbb{Z}_{2}^{\wedge}$ is divisible by $2 a_{k-n}$. Now we can make the following identifications:

$$
\begin{aligned}
\left(\mathbb{S}_{!}^{k} \wedge \mathbf{b o}\right)^{h \mathbb{Z} / 2} & =\operatorname{map}_{\mathbb{Z} / 2}\left(E \mathbb{Z} / 2_{+}, \mathbb{S}_{!}^{k} \wedge \mathbf{b o}\right), \\
\left(\mathbb{S}_{!}^{n} \wedge \mathbf{b o}\right)^{h \mathbb{Z} / 2} & \simeq \operatorname{map}_{\mathbb{Z} / 2}\left(E \mathbb{Z} / 2_{+}, \Omega_{!}^{k-n}\left(\mathbb{S}_{!}^{k} \wedge \mathbf{b o}\right)\right) \\
& \simeq \operatorname{map}_{\mathbb{Z} / 2}\left((E \mathbb{Z} / 2)_{k-n}, \mathbb{S}_{!}^{k} \wedge \mathbf{b o}\right),
\end{aligned}
$$

where $X_{i}:=X / X^{i-1}$ for a CW-space $X$, here $E \mathbb{Z} / 2$ with the standard CW structure having two cells in each dimension, and $\Omega_{!}^{i}$ is the adjoint of $\mathbb{S}_{!}^{i} \wedge$ as a functor from pointed $\mathbb{Z} / 2$-spaces to pointed $\mathbb{Z} / 2$-spaces. (For the last of the identifications, see lemma 8.4 below.) Hence it is enough to show that if an element in

$$
\mathbb{Z} \oplus \mathbb{Z}_{2}^{\wedge} \cong \pi_{k} \mathbf{m a p}_{\mathbb{Z} / 2}\left(E \mathbb{Z} / 2_{+}, \mathbb{S}_{!}^{k} \wedge \mathbf{b o}\right)
$$

lifts to

$$
\pi_{k} \mathbf{m a p}_{\mathbb{Z} / 2}\left((E \mathbb{Z} / 2)_{i}, \mathbb{S}_{!}^{k} \wedge \mathbf{b o}\right)
$$


with $i>0$, then its component in $\mathbb{Z}_{2}^{\wedge}$ is divisible by $2 a_{i}$. Now lemma 8.5 below shows that $\mathbb{S}_{!}^{k} \wedge$ bo can be related to $\mathbb{S}^{k} \wedge$ bo, with the trivial action of $\mathbb{Z} / 2$, by a chain of $\mathbb{Z} / 2$-equivariant maps which are non-equivariant homotopy equivalences. (This uses the hypothesis $4 \mid k$.) Hence it is enough to show that if an element in

$$
\mathbb{Z} \oplus \mathbb{Z}_{2}^{\wedge} \cong \pi_{0} \operatorname{map}\left(B \mathbb{Z} / 2_{+}, \mathbf{b o}\right)
$$

lifts to $\pi_{0} \operatorname{map}\left((B \mathbb{Z} / 2)_{i}\right.$, bo), with $i>0$, then its component in $\mathbb{Z}_{2}^{\wedge}$ is divisible by $2 a_{i}$. But this is well known from Adams' work [Ad2, 7.4] on vector fields on spheres; see also [ABS, §15] and [At].

8.4. Lemma. $\mathbb{S}_{!}^{i} \wedge(E \mathbb{Z} / 2)_{+}$is homeomorphic as a $\mathbb{Z} / 2$-space to $(E \mathbb{Z} / 2)_{i}$.

Proof. We use the standard model for $E \mathbb{Z} / 2$. Then $\mathbb{S}_{!}^{i} \wedge(E \mathbb{Z} / 2)_{+}$is the Thom space of a Whitney sum of $i$ trivial line bundles on $\mathbb{S}^{\infty}$ (each with nontrivial action of $\mathbb{Z} / 2$ covering the antipodal action on the base), and $(E \mathbb{Z} / 2)_{i}=\mathbb{S}^{\infty} / \mathbb{S}^{i-1}$ is the Thom space of $\nu$, the (equivariant) normal bundle of a codimension $i$ sphere $\mathbb{S}^{\infty-i}$ in $\mathbb{S}^{\infty}$. (Think of $\infty$ as a large integer.) But $\nu$ is also a Whitney sum of $i$ trivial line bundles with nontrivial action covering the antipodal action on the base $\mathbb{S}^{\infty-i}$.

8.5. Lemma. If 4 divides $k$, then there exists a $\mathbb{Z} / 2-$ map from $E \mathbb{Z} / 2+\wedge \mathbb{S}_{!}^{k} \wedge$ bo to $\mathbb{S}^{k} \wedge$ bo which is an ordinary homotopy equivalence.

Proof. Since $\mathbb{S}^{k} \wedge$ bo has the trivial action of $\mathbb{Z} / 2$, such a $\mathbb{Z} / 2$-map must factor through the quotient

$$
E \mathbb{Z} / 2_{+} \wedge_{\mathbb{Z} / 2} \mathbb{S}_{!}^{k} \wedge \text { bo }
$$

In fact it is enough to find a map

$$
E \mathbb{Z} / 2_{+} \wedge_{\mathbb{Z} / 2} \mathbb{S}_{!}^{k} \longrightarrow \mathbb{S}^{k} \wedge \mathbf{b o}
$$

whose restriction to the fiber $\mathbb{S}^{k}$ represents a generator of $\pi_{k}\left(\mathbb{S}^{k} \wedge \mathbf{b o}\right) \cong \pi_{0} \mathbf{b o} \cong \mathbb{Z}$, because then we can use the ring structure of bo to extend to $E \mathbb{Z} / 2_{+} \wedge_{\mathbb{Z} / 2} \mathbb{S}_{!}^{k} \wedge \mathbf{b o}$. But now $E \mathbb{Z} / 2_{+} \wedge_{\mathbb{Z} / 2} \mathbb{S}_{\text {! }}^{k}$ is simply the Thom space of $k \eta$, the Whitney sum of $k$ copies of the canonical line bundle $\eta$ on $B \mathbb{Z} / 2$. What we are trying to show is that $k \eta$ has a bo-orientation. Since $k$ is divisible by 4 , the Stiefel-Whitney classes $w_{1}(k \eta)$ and $w_{2}(k \eta)$ vanish. Hence $k \eta$ admits a Spin structure, therefore a $\mathbf{K O}-$ orientation [ABS, Thm.12.3]. For connectivity reasons this lifts to a bo-orientation.

8.6. Calculation. Suppose that $x \in L_{k}^{p}(\mathbb{R} \pi)$ lifts to $L K_{k}(\mathbb{Z} \pi, n)$, where $4 \mid k$ and $4 \mid n$ and $k>n$. Let $V$ be the trivial one-dimensional real representation of $\pi$. Then the $V$-component of the multisignature of $x$ is divisible by $4 a_{k-n}$.

Proof. The $V$-component of the multisignature of $x$ is simply the signature of the image of $x$ under the homomorphism $L_{k}^{p}(\mathbb{R} \pi) \rightarrow L_{k}^{p}(\mathbb{R})$ induced by $\pi \rightarrow\{1\}$. Hence we may assume that $\pi=\{1\}$.

We established divisibility by $2 a_{k-n}$ in 8.3. In fact we shall more or less repeat the argument, and see what improvements we can make. Much as in the proof of 8.3 , we find that it is enough to show that if an element $x$ in

$$
\mathbb{Z} \oplus \mathbb{Z}_{2}^{\wedge} \cong \pi_{k} \mathbf{m a p}_{\mathbb{Z} / 2}\left(E \mathbb{Z} / 2_{+}, \mathbb{S}_{!}^{k} \wedge \mathbf{b o}\right)
$$

lifts to $\pi_{k} \mathbf{m a p}_{\mathbb{Z} / 2}\left((E \mathbb{Z} / 2)_{i}, \mathbb{S}_{!}^{k} \wedge \mathbf{b o}\right)$, and then further to

$$
\pi_{k} \mathbf{m a p}_{\mathbb{Z} / 2}\left((E \mathbb{Z} / 2)_{i}, \mathbb{S}_{!}^{k} \wedge \mathbf{K}(\mathbb{Z})\right)
$$


with $k>i>0$, and $4 \mid i$, then its component $x_{\text {pro }}$ in $\mathbb{Z}_{2}^{\wedge}$ is divisible by $4 a_{i}$. Exactly as in the proof of 8.3 , we see that $x_{\text {pro }}$ is divisible by $2 a_{i}$. (For the present purposes, the best way to see this is to use the Atiyah-Hirzebruch spectral sequence argument which Adams also uses in [Ad1]. At this point it is irrelevant whether we use the Atiyah-Hirzebruch spectral sequence converging to

$$
\pi_{k+*} \operatorname{map}_{\mathbb{Z} / 2}\left(E \mathbb{Z} / 2_{+}, \mathbb{S}_{!}^{k} \wedge \mathbf{b o}\right)
$$

or that converging to $\pi_{*} \mathbf{m a p}\left(B \mathbb{Z} / 2_{+}, \mathbf{b o}\right)$, because they are isomorphic spectral sequences by 8.5. In a moment it will become relevant.) If $x_{\text {pro }}$ is not divisible by $4 a_{i}$, then a nonzero obstruction in $\pi_{k-i}(\mathbf{b o}) \cong \mathbb{Z}$ is responsible for that. The obstruction must lift to a nonzero obstruction in $\pi_{k-i}(\mathbf{K}(\mathbb{Z}))$. However, this is impossible, since $\pi_{k-i}(\mathbf{K}(\mathbb{Z}))$ is a finite group and therefore maps trivially to $\pi_{k-i}(\mathbf{b o}) \cong \mathbb{Z}$.

Remarks. Suppose that $M^{n}$ is a smooth compact manifold, $n \geq 5$, with finite fundamental group $\pi$. There is a well known homomorphism

$$
\pi_{k}\left(\mathbf{L}_{\bullet}^{s}(M)\right) \cong L_{k}^{s}(\mathbb{Z} \pi) \longrightarrow \pi_{k-n-2}(\widetilde{\operatorname{DIFF}}(M) / \operatorname{DIFF}(M))
$$

for any $k \geq n+2$, where $\operatorname{DIFF}(M)$ is the simplicial group of diffeomorphisms $M \rightarrow M$ which agree with the identity on $\partial M$. (A tilde generally signifies "blocks"; in particular

\section{$\widetilde{\mathrm{DIFF}}(M)$}

is the simplicial group whose $k$-simplices are smooth automorphisms of $\Delta^{k} \times M$ restricting to the identity on $\Delta^{k} \times \partial M$, and respecting the faces $d_{i} \Delta^{k} \times M$.) Elements in the kernel of this homomorphism lift to $\pi_{k}(\mathbf{L A} \mathbf{A}(M, n))$, and so their multisignatures (some defined when $2 \mid k$ and all defined when $4 \mid k$ ) must satisfy the divisibility criteria of 8.3 and 8.6. See [WWaut, 6.5] for explanations.

Let $\mathcal{S}\left(\mathbb{S}^{n}\right)$ be the $h$-structure space of $\mathbb{S}^{n}$, again with $n \geq 5$. This can be described as the classifying space of the simplicial groupoid whose objects in degree $k$ are pairs $\left(N^{n}, f\right)$ where $f: N \times \Delta^{k} \rightarrow \mathbb{S}^{n} \times \Delta^{k}$ is a homotopy equivalence over $\Delta^{k}$, and where a morphism from $\left(N_{1}, f_{1}\right)$ to $\left(N_{2}, f_{2}\right)$ is a homeomorphism $g: N_{1} \times \Delta^{k} \rightarrow N_{2} \times \Delta^{k}$ over $\Delta^{k}$ with $f_{2} g=f_{1}$. It is homotopy equivalent to $\mathrm{G}\left(\mathbb{S}^{n}\right) / \mathrm{TOP}\left(\mathbb{S}^{n}\right)$ where $\mathrm{G}\left(\mathbb{S}^{n}\right)$ is the simplicial monoid of homotopy equivalences $\mathbb{S}^{n} \rightarrow \mathbb{S}^{n}$. There is a corresponding block $h$-structure space, which is homotopy equivalent to a component of $\Omega^{\infty} \mathbf{L} \bullet(\mathbb{Z})$, or equivalently, to G / TOP. Elements in

$$
\pi_{k} \tilde{\mathcal{S}}\left(\mathbb{S}^{n}\right) \cong L_{k}(\mathbb{Z}) \cong \pi_{k}\left(\mathbf{L}_{\bullet}(*)\right) \quad(k>0)
$$

which lift to $\pi_{k} \mathcal{S}\left(\mathbb{S}^{n}\right)$ also lift to $\pi_{k}(\mathbf{L} \mathbf{A} \bullet(*, n))$, and so their signatures (defined when $4 \mid k)$ must satisfy the divisibility criterion of 8.6. See [WWaut, 6.4] for explanations.

\section{REFERENCES}

[ABS] M. Atiyah, R. Bott, A. Shapiro, Clifford modules, Topology 3 (1964), 13-38 suppl. MR 29:5250

[Ad1] J. F. Adams, Stable homotopy and generalised homology, Chicago Lectures in Math., University of Chicago Press, 1974. MR 53:6534

[Ad2] J. F. Adams, Vector fields on spheres, Ann. of Math. 75 (1962), 603-632. MR 25:2614

[AdCoDw] A. Adem, R. L. Cohen and W. G. Dwyer, Generalized Tate Homology, Homotopy fixed points, and the transfer, Proc.of 1988 Northw.Univ.Conf.on Alg.Topology, Contemp.Math. 96 (1989). MR 90k:55012 
[At] M. Atiyah, Immersions and imbeddings of manifolds, Topology 1 (1962), 125-132. MR 26:3080

[AtSe] M. Atiyah and G. Segal, Equivariant K-theory and completion, J. Diff. Geom. 3 (1969), 1-18. MR 41:4575

[Car] G. Carlsson, Equivariant stable homotopy and Segal's Burnside ring conjecture, Annals of Math. 120 (1984), 189-224. MR 86f:57036

[Cu] E. B. Curtis, Simplicial homotopy theory, Adv. in Math. 40 (1971), 107-209. MR 43:5529

[GreMa] J. P. C.Greenlees and J.P.May, Generalized Tate, Borel and CoBorel Cohomology, University of Chicago Preprint, 1992.

[Le] D. W. Lewis, Forms over real algebras and the multisignature of a manifold, Advances in Math 23 (1977), 272-284. MR 54:12646

[Lin] W. H. Lin, On conjectures of Mahowald, Segal and Sullivan, Math. Proc. Camb. Phil. Soc. 87 (1980), 449-458. MR 81e:55020

[Ran1] A. Ranicki, The algebraic Theory of Surgery, I, II, Proc.Lond.Math.Soc. (3) 40 (1980), 87-283. MR 82f:57024a; MR 82f:57024b

[Ran2] A. Ranicki, Algebraic L-theory and topological manifolds, Cambridge Tracts in Mathematics, Cambridge Univ.Press, Cambridge, GB, 1992. MR 94i:57051

[Seg] G. Segal, Categories and cohomology theories, Topology 13 (1974), 293-312. MR 50:5782

[TW] L. Taylor and B. Williams, Surgery spaces: formulae and structure, pp. 170-195 in Proc. 1978 Waterloo Algebraic Topology Conf., Lecture Notes in Math. 741, Springer 1979. MR 81k:57034

[Vo] W. Vogell, The involution in the algebraic K-theory of spaces, Proc.of 1983 Rutgers Conf.on Alg. Topology, Springer Lect.Notes in Math.1126 (1985), 277-317. MR 87e:55009

[Wa] C. T. C. Wall, Classification of hermitian forms. VI. Group rings, Ann. of Math. 103 (1976), 1-80. MR 55:5720

[Wald] F. Waldhausen, Algebraic K-theory of Spaces, Proc. of 1983 Rutgers Conf. on Algebraic Topology, Springer Lect.Notes in Math.1126 (1985), 318-419. MR 86m:18011

[WW2] M. Weiss and B. Williams, Automorphisms of Manifolds and Algebraic K-Theory: II, J.Pure and Appl.Algebra 62 (1989), 47-107. MR 91e:57055

[WWaut] M. Weiss and B. Williams, Automorphisms of manifolds, to appear in one of two C.T.C. Wall 60'th Birthday celebration volumes, 1999.

[WWd] M. Weiss and B. Williams, Duality in Waldhausen categories, Forum Math. 10 (1998), 533-603. MR 99g:19002

Department of Mathematics, University of Aberdeen, Aberdeen AB24 3UE, U.K.

E-mail address: m.weiss@maths.abdn.ac.uk

Department of Mathematics, University of Notre Dame, Notre Dame, Indiana 46556

E-mail address: williams.4@nd.edu 